\title{
JET: PROGRESS IN PERFORMANCE AND UNDERSTANDING
}

\author{
The JET Team 1 \\ presented by M. Keilhacker
}

JET Joint Undertaking, Abingdon, Oxon OX14 3EA, UK

\begin{abstract}
In 1990JET operated with a number of technical improvements which led to advances in performance and permitted the carrying out of experiments specifically aimed at improving physics understanding of selected topics relevant to the "NEXT STEP". The new facilities include beryllium antenna screens, a prototype lower hybrid current drive system, and modification of the NI system to enable the injection of ${ }^{3} \mathrm{He}$ and ${ }^{4} \mathrm{He}$. Continued investigation of the hot-ion $\mathrm{H}$-mode produced a value of $\mathrm{n}_{\mathrm{D}}(0) \tau_{\mathrm{E}} \mathrm{T}_{\mathrm{i}}(0)=9 \times 10^{20} \mathrm{~m}^{-3} \mathrm{~s} \mathrm{keV}$, which is near conditions required for $\mathrm{Q}_{\mathrm{DT}}=1$, while a new peaked density profile $\mathrm{H}$-mode was developed with only slightly lower performance. Progress towards steady state operation has been made by achieving ELMy $\mathrm{H}$-modes under certain operating conditions, while maintaining good $\tau_{\mathrm{E}}$ values. Experimental simulation of He ash transport indicates effective removal of alphaparticles from the plasma core for both $\mathrm{L}$ and $\mathrm{H}$ mode plasmas. Detailed analyses of particle and energy transport have helped establish a firmer link between particle and energy transport, and have suggested a connection between reduced energy transport and reversed shear. Numerical and analytic studies of divertor physics carried out for the pumped divertor phase of JET have helped clarify the key parameters governing impurity retention, andan intensive model validation effort has begun. Experimental simulation of alpha-particle effects with $\beta_{\text {tast }}$ up to $8 \%$ have shown that the slowing down processes are classical, and have given no evidence of deleterious collective effects.
\end{abstract}

\section{KEYWORDS}

Tokamak; JET; Fusion Reactor; Particle Transport; Energy Transport; H-mode Physics; Divertor Physics.

\section{INTRODUCTION}

The JET Programme continued during 1990 with the introduction of new machine facilities and the pursuit of its experimental programme of performance optimization at full heating power.

As a result of the positive experience in 1989 with beryllium as the first-wall material (beryllium evaporation onto vessel walls and beryllium tile belt limiters) more plasma facing components were changed to beryllium during the 1989/1990 shut down (Fig. 1). The most successful change, as will be shown, was the replacement of the nickel ICRH antenna screens by beryllium ones. In addition, preliminary beryllium protection tiles were installed in the lower $\mathrm{X}$-point region (graphite tiles were maintained in the upper region), although the shape of these tiles, which had been designed originally for a different purpose, was not optimised for high power fluxes to the $\mathrm{X}$-point.

The other main new facilities introduced for the 1990 experimental campaign were the prototype lower hybrid current drive (LHCD) launcher and the conversion of one of the two neutral beam systems to operate either at $140 \mathrm{keV}$ in deuterium or at $120 \mathrm{keV}$ in helium. In the LHCD experiments up to $1.6 \mathrm{MW}$ of LH power was coupled to the plasma. With preheating by Ion Cyclotron Resonance Heating (ICRH) with a power of $\sim 4 \mathrm{MW}$, non-inductive currents up to $1.2 \mathrm{MA}$ were driven and a current drive efficiency $\gamma \sim 0.4 \times 10^{20} \mathrm{~m}^{-2} \mathrm{~A} / \mathrm{W}$ was

${ }^{1}$ See Appendix 1 
achieved. (Here $\gamma$ is defined as $\gamma=\tilde{n}_{e} R I / P_{C D}$, where $P_{C D}=P_{L H}+I_{C D} V_{\text {loop }}$ and $\tilde{n}_{e}$ is the line average plasma density). Helium neutral beam injection was carried out with up to $5 \mathrm{MW}$ of ${ }^{3} \mathrm{He}$ and $7 \mathrm{MW}$ of ${ }^{4} \mathrm{He}$. The use of helium beams is interesting because it significantly reduces both the neutron yield and the consequent vessel activation and allows a more direct interpretation of measurements of neutron yields from thermal plasma reactions. Finally, and most importantly, helium injection provides a precise particle source for simulation studies of alpha-particle transport and for ion cyclotron minority heating.

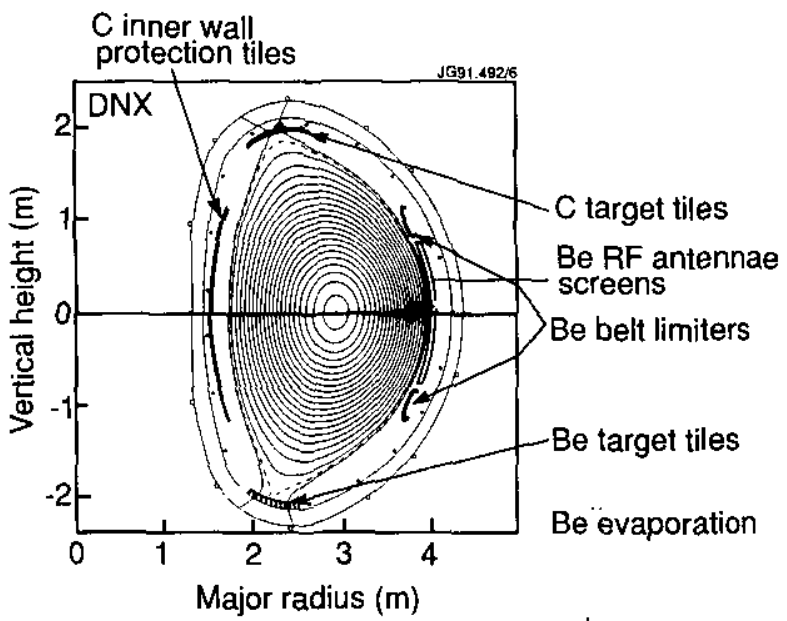

Fig. 1: Plasma facing components in 1990 campaign.

With the help of these new facilities good progress was made again during the last year in improving plasma performance and physics understanding. In hot-ion $\mathrm{H}$-mode discharges with a central ion temperature of $\mathrm{T}_{\mathrm{i}}(0)=$ $28 \mathrm{keV}$, a fusion triple product $\mathrm{n}_{\mathrm{D}}(0) \tau_{\mathrm{E}} \mathrm{T}_{\mathrm{i}}(0) \sim 9 \times 10^{20} \mathrm{~m}^{-3} \mathrm{~s} \mathrm{keV}$ has been achieved, while in pellet fuelled $\mathrm{H}$-mode discharges with peaked density profiles and roughly equal electron and ion temperatures $\mathrm{T}_{\mathrm{e}}(0) \sim \mathrm{T}_{\mathrm{i}}(0) \sim 10 \mathrm{keV}$

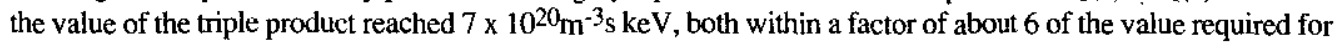
ignition in a D.T fusion reactor.

This paper gives an overview of JET results from the 1990 campaign and the ensuing analysis. It is divided into two main themes. First, Section 2 reports improvements of plasma performance in high current limiter and X-point discharges. This is followed in Section 3 by a discussion of progress in understanding the underlying tokamak physics in four areas which have been selected because of their relevance to NEXT STEP/Reactor issues. The subjects discussed are particle and energy transport, H-mode physics and the approach to steady state, divertor physics and impurity control, and the experimental simulation of $\alpha$-particle effects. Section 4 concludes with a summary of the results and their implications for a NEXT STEP/Reactor.

This paper is complemented by another invited JET paper [The JET Team, presented by Jacquinot, 1991] which concentrates on ICRH and LHCD results. In addition, details of many of the topics discussed here can be found in contributed JET papers presented at this conference, as referenced.

\section{IMPROVEMENT OF PLASMA PERFORMANCE}

Improvements in plasma performance have been achieved both in limiter and X-point geometry. Plasma currents of 7 MA have been sustained for $3 \mathrm{~s}$ using the beryllium belt limiters. Two modes of X-point operation have been further developed. In the low density, hot ion H-mode, central ion temperatures of about $30 \mathrm{keV}$ are obtained. A different $\mathrm{H}$-mode has been developed in which higher density, sharply peaked profiles are produced, with approximately equal electron and ion temperatures in the range of $10 \mathrm{keV}$. These $\mathrm{X}$-point modes have produced plasmas with "QDT equivalent" values between 0.4 and 0.9 , albeit only transiently. 


\subsection{High Current Limiter Plasmas}

The optimization of fusion performance in limiter discharges requires operation at the highest plasma currents compatible with technical and $q$-limit constraints while at the same time trying to maintain peaked density and temperature profiles.

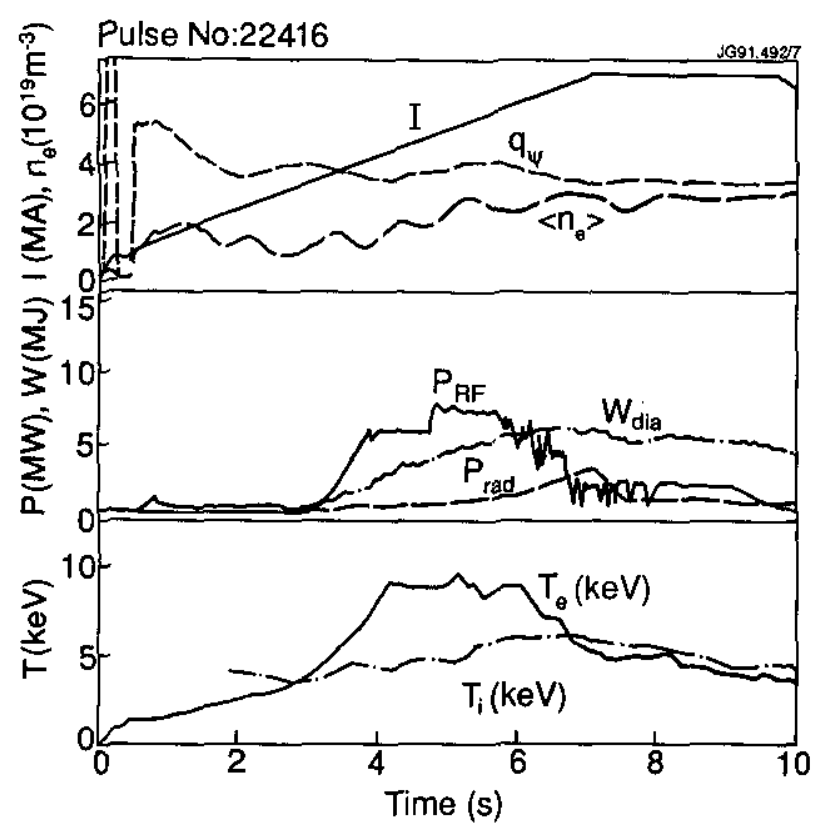

Fig. 2: Various time traces for a 7 MA limiter discharge with a 3 sflat-top (Pulse No: 224l6) on the beryllium belt.

During the 1990 experimental campaign, operation at 7 MA was demonstrated for the first time on the beryllium belt limiter [Lomas et al., 1991]. A number of technical improvements allowed the development of a strong plasma cross-section expansion scenario (Fig. 2 ) in which $\mathrm{q}_{\psi}=4$ could be passed early in the current ramp. Thereafter, $\mathrm{q}_{\psi}$ was kept between 3 and 4 by programming the minor radius, elongation and triangularity. This scenario allowed a fast $1 \mathrm{MA} / \mathrm{s}$ current rise to $7 \mathrm{MA}$ and was very economical in flux consumption, with $8 \mathrm{~V}$-s remaining after a flat top of 3s. During the course of these experiments the machine survived two disruptions at $7 \mathrm{MA}$ in which plasma motion induced poloidal currents up to $1 \mathrm{MA}$ in the vessel giving rise to a vertical force of 350 connes.

The application of a few megawatts of ICRF heating during the current rise phase suppressed sawteeth for plasma currents up to $6 \mathrm{MA}$. (A sawtooth-free period of $8 \mathrm{~s}$ was obtained in a 3 MA discharge.) Examination of sawtooth free discharges at $5 \mathrm{MA}$ shows that the electron temperature increases approximately linearly with ICRH power per particle up to $\mathrm{P}_{\mathrm{RF}} / \mathrm{n}_{\mathrm{e}}(0) \sim 3 \times 10^{-19} \mathrm{MWm}^{3}$ but remains at $\mathrm{T}_{\mathrm{e}}(0)=12-13 \mathrm{keV}$ for $\mathrm{P}_{\mathrm{RF}} / \mathrm{n}_{\mathrm{e}}(0)$ in the range $3-6 \times 10^{-19} \mathrm{MWm}^{3}$. According to [Cordey et al., 1991] this apparent saturation of the electron temperature with ICRF power is caused by a saturation of the central heating power density and is not a transport phenomenon. A significant part of the broadening of the heating profile can be accounted for by fast ion finite orbit effects.

In order to increase the ion temperature in these plasmas, Neutral Beam Injection (NBI) was added to the RF heating. At $6.5 \mathrm{MA}, 6 \mathrm{MW}$ of ICRF combined with $8 \mathrm{MW}$ of NBI produced a sawtooth free period of $0.8 \mathrm{~s}$ with $\mathrm{T}_{\mathrm{e}}(0) \sim 8.0$ $\mathrm{keV}, \mathrm{T}_{\mathrm{i}}(0) \sim 7.5 \mathrm{keV}, \mathrm{n}_{\mathrm{e}}(0) \sim 5 \times 10^{19} \mathrm{~m}^{-3}$ and $\tau_{\mathrm{E}} \sim 0.65 \mathrm{~s}$. The central fuel concentration was $\mathrm{n}_{\mathrm{D}}(0) / \mathrm{n}_{\mathrm{e}}(0) \sim 0.88$ giving ${ }_{\mathrm{D}}(0) \tau_{\mathrm{E}} \mathrm{T}_{\mathrm{i}}(0) \sim 2.1 \times 10^{20} \mathrm{~m}^{-3} \mathrm{~s} \mathrm{keV}$ (see Table I). The confinement time supports a linear scaling with plasma current in sawtooth - free discharges, but data at the highest currents is still limited in additional heating power. 
Table I: Comparison of High Performance Plasmas

\begin{tabular}{l|c|c|c|c} 
Configuration & $\begin{array}{c}7 \text { MA L-Mode } \\
\text { Beryllium } \\
\text { Belt Limiter }\end{array}$ & $\begin{array}{c}\text { Hot-Ion H-Mode } \\
\text { Single Null } \\
\text { X-Point }\end{array}$ & \multicolumn{2}{|c}{$\begin{array}{c}\text { PEP H-Mode } \\
\text { Double Null } \\
\text { X-point }\end{array}$} \\
\hline Heating & NBI + ICRH & NBI & NBI & ICRH \\
\hline Pulse No & 22410 & 22689 & 22624 & 22490 \\
$\mathrm{I}_{\mathrm{p}}(\mathrm{MA})$ & 6.5 & 3.6 & 3.6 & 3.0 \\
$\mathrm{~B}_{\mathrm{T}}(\mathrm{T})$ & 3.4 & 2.8 & 3.3 & 2.8 \\
$\mathrm{P}_{\text {tot }}(\mathrm{MW})$ & 15 & 18 & 13 & 12 \\
$\mathrm{~W}_{\text {dia }}(\mathrm{MJ})$ & 7.5 & 11.3 & 6.7 & 8.3 \\
$\mathrm{~T}_{\mathrm{e}}(0)(\mathrm{keV})$ & 8.0 & 10.0 & 8.3 & 10.0 \\
$\mathrm{~T}_{\mathrm{i}}(0)(\mathrm{keV})$ & 7.5 & 28 & 9.8 & 9.5 \\
$\mathrm{n}_{\mathrm{D}}(0)\left(\mathrm{m}^{-3}\right)$ & $4.4 \times 10^{19}$ & $4.0 \times 10^{19}$ & $6.5 \times 10^{19}$ & $8 \times 10^{19}$ \\
$\tau_{\mathrm{E}}(\mathrm{s})$ & 0.65 & 0.85 & 0.73 & 1.0 \\
$\mathrm{n}_{\mathrm{D}}(0) \mathrm{\tau}_{\mathrm{E}} \mathrm{T}_{i}(0)\left(\mathrm{m}^{-3} \mathrm{skeV}\right)$ & $2.1 \times 10^{20}$ & $9.5 \times 10^{20}$ & $4.7 \times 10^{20}$ & $7.8 \times 10^{20}$ \\
$\mathrm{R}_{\mathrm{DD}}\left(\mathrm{s}^{-1}\right)$ & $0.7 \times 10^{16}$ & $7.6 \times 10^{16}$ & $4.1 \times 10^{16}$ & $2.0 \times 10^{16}$ \\
$\mathrm{QDD}_{\mathrm{DD}}$ & $2.8 \times 10^{-4}$ & $2.8 \times 10^{-3}$ & $1.8 \times 10^{-3}$ & $1.5 \times 10^{-3}$ \\
$\mathrm{QDT}_{\mathrm{DT}}$ & - & $0.8-0.9$ & $0.4-0.5$ & $0.3-0.4$
\end{tabular}

\subsection{High Performance X-Point Plasmas}

\section{a) Hot ion H-mode}

The development of the 'hot-ion H-mode' [Watkins et al., 1989, Balet et al., 1990, and Keilhacker and the JET Team, 1990] has allowed the attainment in JET of plasma conditions close to those required for QDT 1. However, these conditions have, to date, been transient and their duration has been limited by the occurrence of a strong influx

Pulse No: 22689

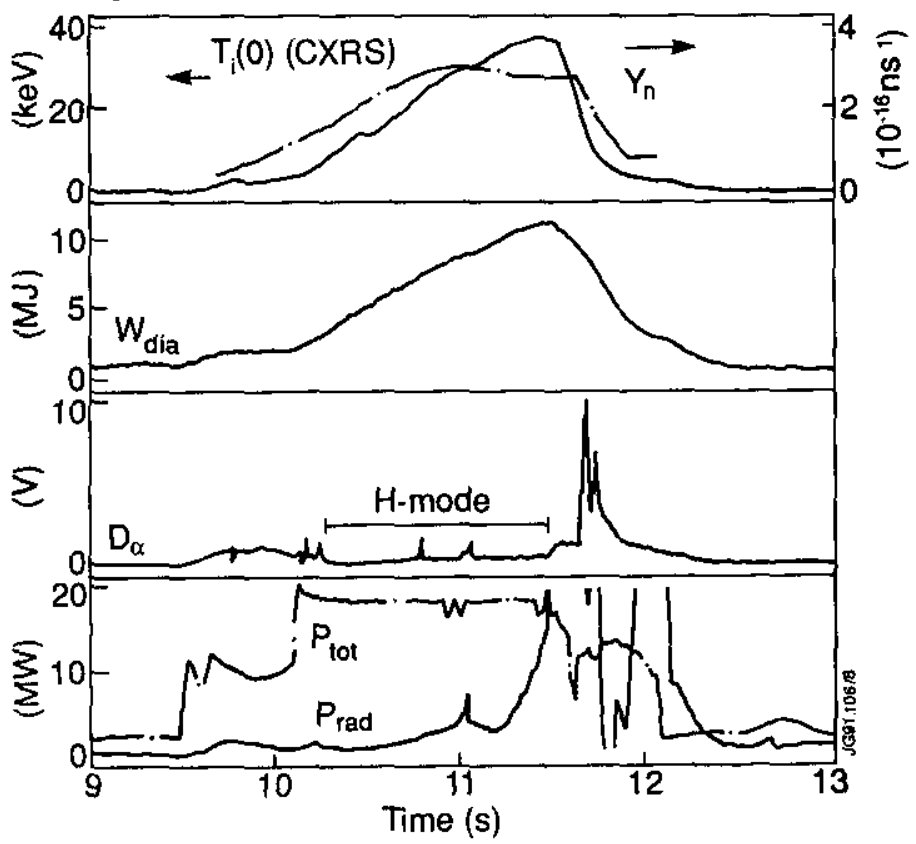

Fig. 3: Various time traces for a hot-ion H-mode plasma (Pulse No: 22689, 3.6MA/2.8T, "reversed" toroidal magnetic field). 
of carbon (dubbed "carbon catastrophe"). A major advance in our effort to extend this regime in duration and performance was achieved last year by exploiting the more uniform deposition of power on the inner and outer divertor target plates when operating with "reversed toroidal field", i.e. with the ion $\nabla$ B-drift away from the X-point (this is discussed in more detail in Section 3.2.b). The duration of the regime has been extended to 1.2s without $\mathrm{X}$ point sweeping and to $1.5 \mathrm{~s}$ when combined with 'vertical' sweeping of the $\mathrm{X}$-point to target distance, $\Delta \mathrm{X}$. Since impurity shielding appeared to be improved at larger $X$-point to target distances $(\Delta X \sim 10 \mathrm{~cm})$, high current $\left(3 \leq I_{p}\right.$ $\leq 4 \mathrm{MA}$ ) single-null $\mathrm{X}$-point plasmas were used in preference to the double-null $\mathrm{X}$-point configuration which is more limited in the $\Delta \mathrm{X}$ values that can be obtained.

Figure 3 illustrates the time evolution of the pulse with the highest neutron production and best value of the fusion triple product ${ }_{\mathrm{D}}(0) \tau_{\mathrm{E}} \mathrm{T}_{\mathrm{i}}(0)$. In this $3.6 \mathrm{MA} / 2.8 \mathrm{~T}$ discharge with $18 \mathrm{MW}$ of neutral beam injection $(10 \mathrm{MW} \mathrm{of} 80 \mathrm{keV}$ $\mathrm{D}^{\circ}$ and $8 \mathrm{MW}$ of $140 \mathrm{keV} \mathrm{D}$ ) central ion and electron temperatures of $\mathrm{T}_{\mathrm{i}}(0) \sim 28 \mathrm{keV}$ and $\mathrm{T}_{\mathrm{e}}(0) \sim 10 \mathrm{keV}$ were achieved (see Table I). The value of $\mathrm{n}_{\mathrm{D}}(0) \mathrm{te}_{\mathrm{E}} \mathrm{T} ;(0) \sim 9-10 \times 10^{20} \mathrm{~m}^{-3} \mathrm{~s} \mathrm{keV}$ was slightly higher than the previous record, as was the fusion reaction rate of $7.6 \times 10^{16} \mathrm{~s}^{-1}$, corresponding to a QDD of $2.8 \times 10^{-3}$. In addition, QDD $>$ $2.0 \times 10^{-3}$ was maintained for over $0.5 \mathrm{~s}$. A detailed analysis, using the TRANSP code, of a similar pulse ( $4 \mathrm{MA} /$ $2.8 \mathrm{~T}$ ) with almost the same fusion reaction rate shows that while these discharges are initially dominated by beamthermal reactions, when the peak fusion reactivity is reached thermal reactions account for $\sim 50 \%$ of the fusion power (Fig. 4) [Stubberfield et al., 1991].

Hot-ion H-modes are characterized by a significant population of fast, beam injected ions, which contribute not only to the reaction rate, but also to the plasma energy. In addition, both density and ion temperature profiles are quite peaked. Energy confinement of these discharges is very good and can reach three times the Goldston L-mode value.

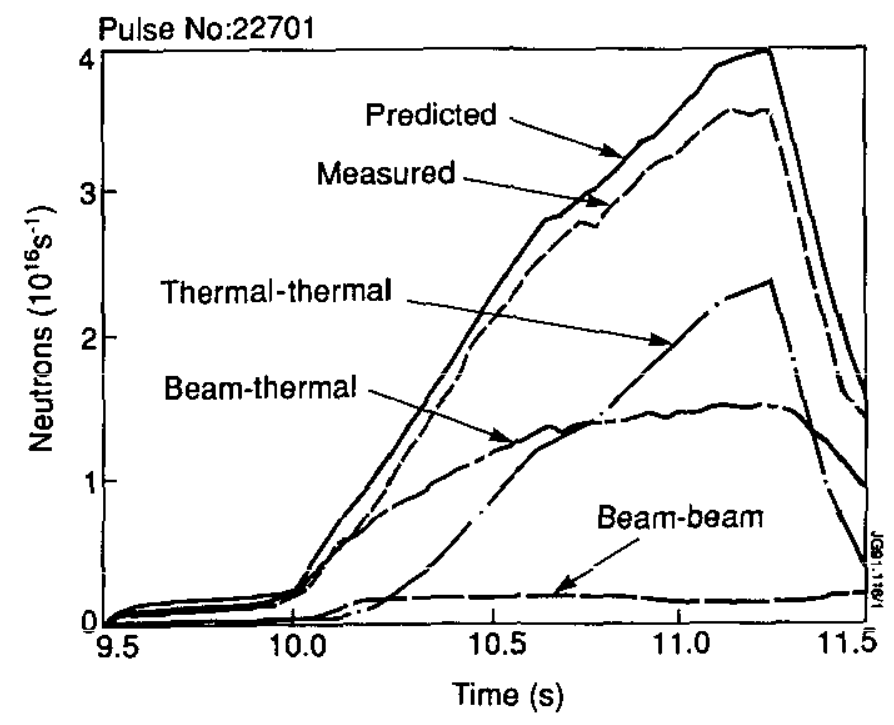

Fig. 4: Contributions to neutron rate for an H-mode pulse (Pulse No: 22701 , 4MA/2.8T) similar to that shown in Fig. 3.

\section{b) Pellet-peaked density profile H-mode}

The second route to high fusion yield tries to combine the good confinement properties of the H-mode, which normally has quite flat profiles, with peaked profiles produced by pellet fuelling and central heating, i.e. with the pellet enhanced performance (PEP) mode previously observed in limiter discharges [Schmidt and the JET Team, 1989].These experiments [Tubbing et al., 1991, and Kupschus et al., 1991] were carried out in double null X-point configuration, with plasma currents in the range 3.0 to $3.6 \mathrm{MA}$ and toroidal fields in the range 2.8 to $3.2 \mathrm{~T}$. The central heating was in most cases supplied by ICRH (with $\mathrm{H}$ or ${ }^{3} \mathrm{He}$ minority) but similar results were also achieved with NBI. For a PEPmode todevelop, the pellet has tobe injected well before the onset of sawteeth, which in turn requires 
that the X-point configuration is formed early in the discharge. An example of a PEP H-mode with NBI heating is shown in Fig. 5 where two $2.7 \mathrm{~mm}$ pellets and a $4 \mathrm{~mm}$ pellet were injected at $t=4.5,4.75$ and $5.0 \mathrm{~s}$, the third pellet raising the central plasma density to $1.2 \times 10^{20} \mathrm{~m}^{-3}$. At $5.0 \mathrm{~s}, 10.6 \mathrm{MW}$ of NBI (7.8 MW at 140 $\mathrm{kV}$ plus $2.8 \mathrm{MW}$ at $80 \mathrm{kV}$ ) and $\sim 2 \mathrm{MW}$ of $\mathrm{lCRH}$ were switched on and a slow transition to $\mathrm{H}$-mode occurred.The neutron rate reaches a maximum of $2 \times 10^{16} \mathrm{~s}^{-1}$ at $6.0 \mathrm{~s}$. At this time, the central ion and electron temperatures are very similar, $T_{i}(0)=9.8 \mathrm{keV}$ and $T_{e}(0)=8.3 \mathrm{keV}$, the central deuterium density is $n_{D}(0)$ $=6.5 \times 10^{19} \mathrm{fl}^{-3}$ and the energy confinement time is $\tau_{E}=0.73 \mathrm{~s}$, resulting in a fusion product $\mathrm{n}_{D}(0) \tau_{E} \mathrm{~T}_{i}(0)$

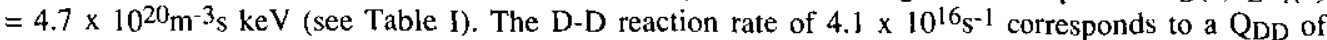
$1.8 \times 10^{-3}$. In a similar pulse with mainly ICRF heating (Pulse No: 22490 , Table I) the product $\mathrm{n}_{\mathrm{D}}(0) \tau_{\mathrm{E}} \mathrm{T}_{\mathrm{i}}(0)$ is $7.8 \times 10^{20} \mathrm{~m}^{-3} \mathrm{~s} \mathrm{keV}$, but the fusion reaction rate is a factor 2 down (fewer non-thermal neutrons).

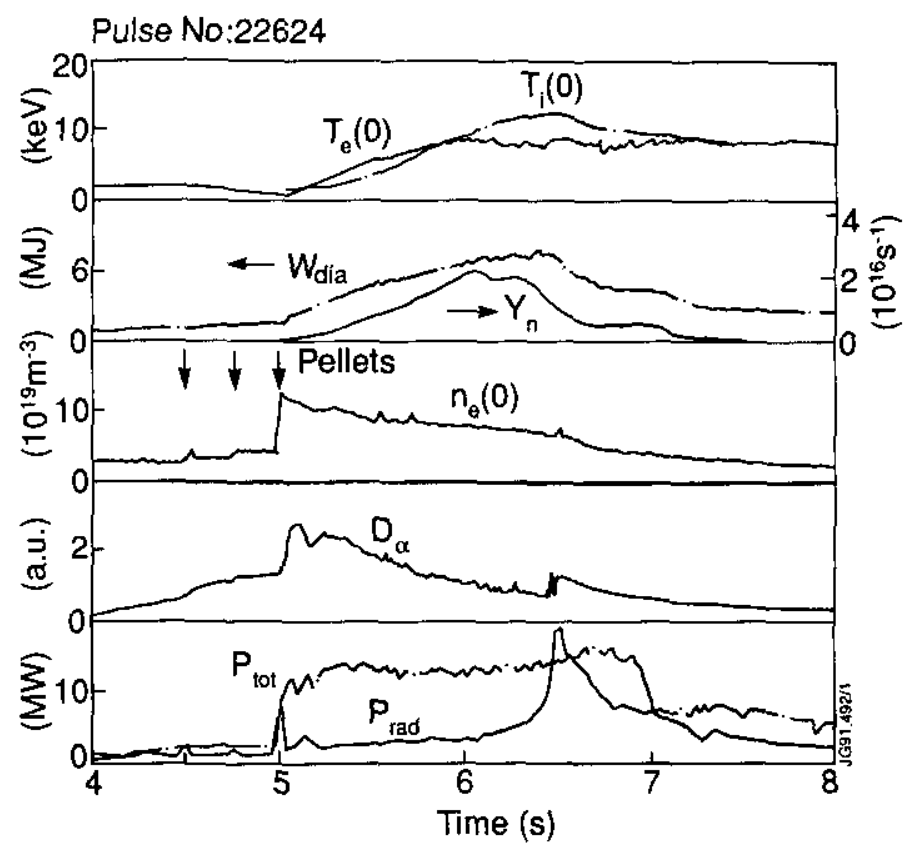

Fig. 5: Various time traces for an H-mode plasma with pellet-peaked density profile (Pulse No: 22624,3.6MA/3.3T)

A D-D simulation using the TRANSP code for this pulse showed about equal contributions from beamthermal and thermal-thermal reactions to the neutron yield at peak fusion reactivity, while in similar plasmas with ICRF heating thermal reactions account for $\sim 80 \%$ of the fusion yield. D-T simulations of PEP H-mode Pulse No: 22624 using various scenarios for beams, pellets, plasma composition and particle transport yield equivalent QDT values between 0.4 and 0.5, while for the hot ion H-mode (Pulse No: 22689), QDT values of 0.8-0.9 are obtained [Stubberfield et al., 1991].

In these discharges, the pressure gradient is very high in the plasma core, exceeding the local value of $j_{\phi} B_{\theta}$ (toroidal current density multiplied by poloidal field). The resulting calculated bootstrap current density can become as large locally as the pre-existing ohmic current density $\left(\sim 1 \mathrm{MA} \mathrm{m}^{-2}\right)$ which should drive the toroidal electric field close to zero in this region. The high bootstrap current region at around $1 / 3$ of the plasma radius has a width of $\sim 0.2 \mathrm{~m}$ and persists for approximately $1 \mathrm{~s}$. As resistive diffusion restores the electric field, the cursent in the high bootstrap region increases, leading to an off axis peak in the current density and to a region of negative shear. The time required for this to evolve completely is also $\sim 1$ s (at 5 $\mathrm{keV}$ ) but a negative shear can occur on a shorter timescale if the shear is initially small in the plasma core. It is believed that this shear reversal may play a role in the improved confinement (see Sect.3.1.b)). 
The stability of these pellet enhanced configurations has been analysed. The high central pressures obtained are such that the value of the quantity $\beta_{p}$ which occurs in the theory of $m=1$ modes [Bussac et al., 1975] is substantially greater than 1.0 . If there is a $q=1$ surface in the plasma such a high $\beta_{\mathrm{p}}$ is predicted to be strongly unstable to the $m=l$ ideal mode, and the inference must be that, at the time of these high pressures, $q$ is greater than unity everywhere. This is consistent with the bootstrap current calculations if the q-profile has an off-axis minimum with $\mathrm{q}_{\min }>1$.

This form of q-profile also has implications for ballooning modes. With a normal q-profile, a high pressure gradient in the region of low shear is vulnerable to ballooning instabilities, but the region of negative shear inside the radius of minimum $q$ is predicted to be stable to ballooning modes. The overall situation is therefore that, although the plasma pressure is very high, the most threatening instabilities are avoided. However, as current diffusion progresses a number of MHD instabilities do arise and appear to be the cause of the deterioration of this enhanced mode of operation. Observations of the structure and position of these instabilities are further evidence for negative shear in the centre [Smeulders et al., 1991].

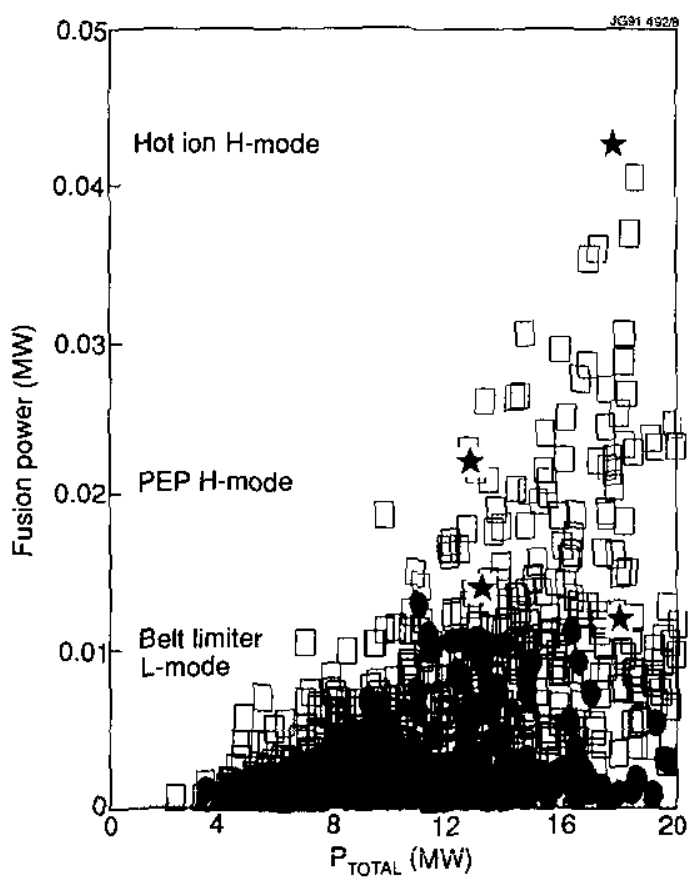

Fig. 6: D-D fusion power versus total heating power for discharges from the $1986-90$ period.

Table I shows a comparative summary of typical plasma parameters that have been achieved in the three high performance modes of operation discussed above. The performance aspect is also illustrated in Fig. 6, where the D-D fusion power is plotted versus total input power for a large number of shots assembled during the 1986-90 period, with the "best" shots listed in Table I specially marked. For discharges with ICRF heating (full symbols) the fusion yield originates mainly from thermal reactions while for the NB heated discharges (open symbols) there is a considerable contribution from beam-plasma reactions (c.f. Fig. 4). It is worth noting that if the strong influx of carbon could be controlled (or at least delayed) at high heating powers (in particular also for $P_{\text {tot }}>20 \mathrm{MW}$ ), the fusion power would considerably exceed the highest presently achieved values. To conclude the discussion on performance it should also be mentioned that in ICRH experiments with $\mathrm{D}\left({ }^{3} \mathrm{He}\right)$ minority heating a fusion yield of $140 \mathrm{~kW}$ has been achieved with an $R F$ power of $10-14 \mathrm{MW}$ giving $\mathrm{QD}^{3} \mathrm{He} \geq 1 \%$. 


\section{IMPROVED PHYSICS UNDERSTANDING}

In the following, four key areas of tokamak physics are discussed in which recent JET work has improved our understanding and implications for a NEXT STEP and a tokamak fusion reactor are pointed out. The four areas selected are particle and energy transport, $\mathrm{H}$-mode physics and the approach to steady state, impurity retention in the divertor, and experimental simulation of the behaviour of alpha-particles in an ignited plasma.

\subsection{Particle and Energy Transport}

In this section, recent JET work in the area of tokamak transport is highlighted: the investigation of particle and impurity transport using transient events, the analysis of local energy transport using interpretative and predictive simulation codes, and finally heat and particle pulse propagation experiments including a discussion of the difference between dynamic (heat pulse) and static (power balance) transport measurements.

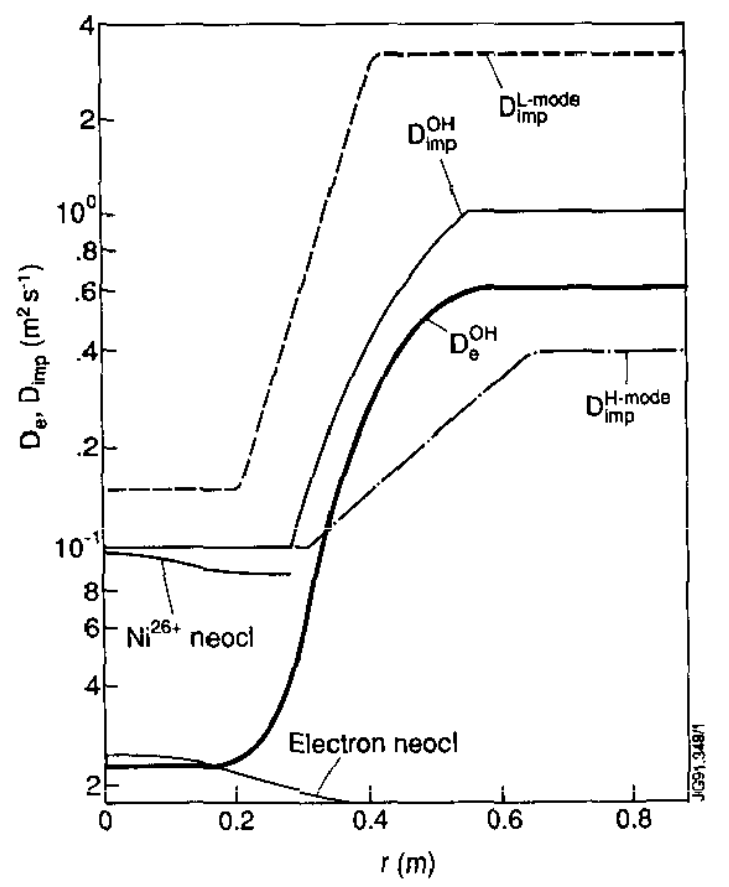

Fig. 7: Radial dependence of diffusion coefficients of electrons (for $O H$ ) and impurities (for $O H, L$-and $H$-mode). Neoclassical levels at the centre are also indicated for comparison.

\section{a) Particle and impurity transport}

To separate the contributions of diffusion and convection to particle transport, it is necessary to investigate transient events with high space and time resolution. In JET, an analysis of the time evolution of electron and impurity (Ni) density profiles in ohmically heated discharges (see Fig. 7) shows that within a small volume $(\mathrm{r} / \mathrm{a} \leq 0.3)$ near the magnetic axis the particle diffusion coefficients are strongly reduced $\left(D_{e}-0.02 \mathrm{~m}^{2} \mathrm{~s}^{-1}, \mathrm{D}_{\text {imp }} \sim 0.1 \mathrm{~m}^{2} \mathrm{~s}^{-1}\right)$ in comparison with their value at larger radii $\left(\mathrm{D}_{\mathrm{e}} \sim 0.6 \mathrm{~m}^{2} \mathrm{~s}^{-1}, \mathrm{D}_{\text {imp }} \sim 1.0 \mathrm{~m}^{2} \mathrm{~s}^{-1}\right)$ with a rather abrupt change between the two regions. This is also seen in L-mode discharges and, although with a smoother transition, in $\mathrm{H}$-mode discharges. This result is quite general for impurities: it has been demonstrated by laser blow-off experiments in ohmic, L-mode, and $\mathrm{H}$-ntode discharges [Pasini et al., 1991] and by the evolution of intrinsic impurity profiles following pellet injection [Behringer et al., 1988]. For electrons it has so far only been demonstrated in (i) the 
sawtooth-free phases following pellet injection and(ii) in the current rise preceding the onset of sawteeth [Giannella et al., 1991]. Sawteeth "short-circuit" the inner (good) and outer (poor) confinement regions.

It appears, then, that within the central region $(\mathrm{r} / \mathrm{a} \leq 0.3)$ the particle transport is neoclassical in many cases. The observed peaking of the electron density profile is reproduced by the neo-classical (Ware) pinch, whilst the impurity diffusion coefficient $\left(\mathrm{D}_{\mathrm{imp}}=0.10,0.15\right.$ and $0.10 \mathrm{~m}^{2} \mathrm{~s}^{-1}$, for $\mathrm{OH}, \mathrm{L}$ - and $\mathrm{H}$-mode discharges respectively) is close to the neoclassical value. At larger radii $(r / a=0.4-0.8)$, however, neither the electron source distribution (due to the ionization of edge neutrals) nor the Ware pinch are sufficient to account for the observed density gradients, unless a substantial reduction of the diffusion coefficient below the level inferred from transients (see above) is postulated. One mechanism for an anomalous particle pinch that would sustain such gradients, which has been proposed by Rebut and co-workers [Boucher et al., 1991], arises from parallel thermal forces between impurity and deuterium ions in a plasma with partly chaotic magnetic topology.

In ELM-freeH-mode discharges, impurity transport is reduced in the external region compared to L-mode discharges. To model these discharges requires a large, very localised convective velocity near the separatrix. Impurity confinement times for these discharges aretypically of orderseveral seconds, much longer than the respectiveenergy confinement times. (This is in contrast to the L-mode regime where roughly equal particle and energy confinement times are observed).

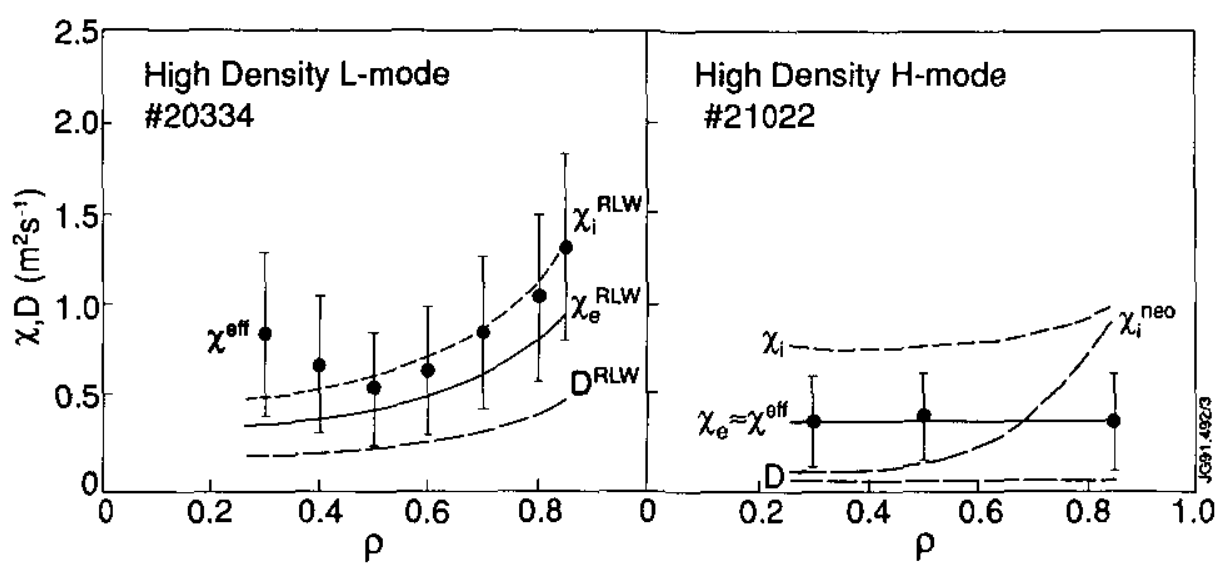

Fig. 8: Comparison of modelled electron thermal, ion thermal and hydrogenic particle conductivities $\chi_{\mathrm{r}}, \chi_{\mathrm{i}}$ and $D$ versus flux coordinate $\rho$ for a high density $L$-and $H$-mode case. The bars represent the range of the "experimental" $\chi^{\text {tf }}$.

\section{b) Energy transport in improved confinement regimes}

Here we discuss the analysis of local energy transport in improved confinement regimes such as H-mode and PEP $\mathrm{H}$-mode, using interpretative and predictive simulation codes.

In a study using the predictive equilibrium - transport code JETTO, the transport properties of high density $\left(\left\langle n_{e}\right\rangle\right.$ $\sim 5 \times 10^{19} \mathrm{~m}^{-3}$ ) L (limiter)- and $\mathrm{H}$-mode discharges with the same plasma current (3.1 MA) and neutral beam heating power (7.5 MW) were compared [Taroni, Sack et al., 1991]. The critical electron temperature gradient model by Rebut, Lallia and Watkins (RLW model) [Rebut et al., 1989], modified in the external region (r/a $\geq 0.75$ ) as described in [Taroni $e t$ al., 1991], simulates well the temperature profiles in both the L- and H-modes. The corresponding profiles of $\chi_{\mathrm{e}}$ and $\chi_{\mathrm{i}}$ are shown in Fig. 8 . In the central region of the plasma $(\mathrm{r} / \mathrm{a} \leq 0.6)$ the high density limiter L-mode shows low values of $\chi_{e}$ and $\chi_{i}$, comparable to those in the $H$-mode discharge. In the L-mode case, however, $\chi_{\mathrm{e}}$ and $\chi_{\mathrm{i}}$ increase in the region $\mathrm{r} / \mathrm{a} \geq 0.6-0.7$, while low $\chi_{\mathrm{e}}$ and $\chi_{\mathrm{i}}$ values are maintained in this region in the $\mathrm{H}$-mode. These results are in agreement with values of $\chi^{\mathrm{eff}}=-\mathrm{q} / 2 \mathrm{n}_{\mathrm{e}} \nabla \mathrm{T}_{\mathrm{e}}$, where $\mathrm{q}$ is the total heat flux, obtained by the interpretive code FALCON from experimental rather thar "predicted" profiles.

The comparison shows that in the bulk plasma region $(\mathrm{r} / \mathrm{a} \leq 0.7)$ the local transport coefficients have comparable values in $\mathrm{L}$ - and $\mathrm{H}$-mode discharges with similar density. The improvement in the $\mathrm{H}$-regime is mainly due to the 
lower values of local transport coefficients at the plasma edge which are similar to or even lower than those in the ohmic phase. Other simulations indicate that at the $\mathrm{L}$ to $\mathrm{H}$ transition a rapid reduction in transport takes place at the very edge of the plasma which gradually extends over the whole plasma volume on a global confinement time scale, mainly as a result of changes in the bulk plasma parameters (e.g. increase in density [Watkins et al., 1989]). They also show that $\chi_{\mathrm{i}}$ is close to neoclassical levels in the central region of the best JET H-mode discharges (hot-ion and PEP regimes) and that, in general, $\chi_{i}>\chi_{\mathrm{e}}$ in the outer plasma region (in cases where both can be determined).

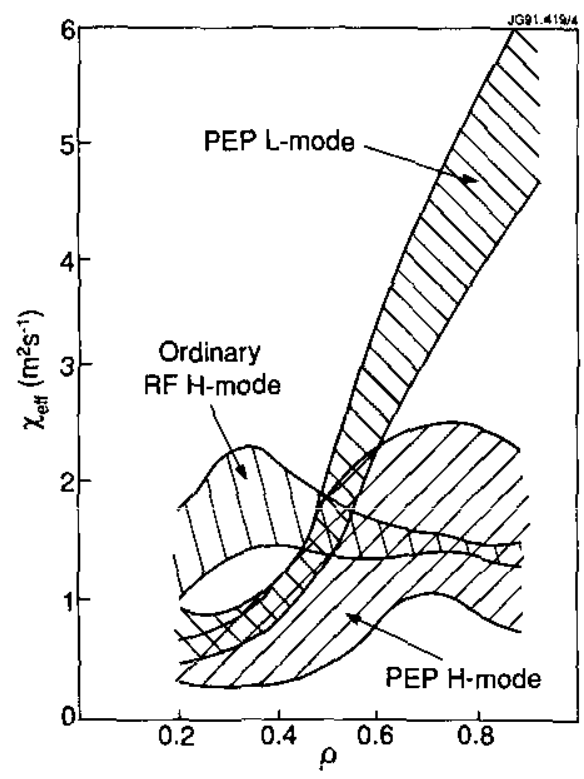

Fig. 9: Comparison of transport in "normal" $H$-mode with PEP L-and PEP H-modes

The predictive code was also used to model the enhanced performance of $\mathrm{L}$ - and $\mathrm{H}$-mode discharges with pellet injection (so-called PEP-mode). Since interpretative transport studies show improved confinement in the plasma core (see Fig. 9), where there are strong indications of negative shear (see Sect. $2.2 \mathrm{~b}$ )), the simulations were carried out by reducing transport to the neoclassical levels in regions where $\mathrm{dq} / \mathrm{dr}$ is negative, as in the RLW model. The simulations (for PEP L-mode Pulse No: 17749) show that, within the uncertainties related to the evaluation of the ICRH power deposition profiles, $\chi_{\mathrm{i}}$ is neoclassical, and $\chi_{\mathrm{e}}$ is close to $\chi_{\mathrm{i}}$ (and not neoclassical as in the RLW model). As an example of this simulation, Fig.10 compares computed $\mathrm{T}_{\mathrm{e}}$-profiles with $\left(\mathrm{T}_{\mathrm{e}}{ }^{\text {comp(1) }}\right)$ and without $\left(\mathrm{T}_{\mathrm{e}}{ }^{\text {comp(2) }}\right)$ the described reduction of heat transport $\left(\chi_{\mathrm{e}} \sim\right.$ $\left.\chi_{i}=\chi_{i, n e o}\right)$ with measured $T_{e}$-profiles $\left(T_{e} e^{L I D A R}\right)$. The simulation using the reduced ion transport not only reproduces well the measured $\mathrm{T}_{\mathrm{e}}$-profile but also shows the required outward shift of the magnetic axis resulting from the relatively high central pressure and low central current density (the latter is mainly an effect of the off-axis bootstrap current). In the region of non-inverted q-profile, energy transport is consistent with the RLW model and similar to standard L-mode discharges. Preliminary results of particle transport analysis show that $D$ is also low $\left(\leq 0.1 \mathrm{~m}^{2} \mathrm{~s}^{-1}\right)$ in the central region.

The results thus suggest that the q-profile might play an important role in determining the local transport coefficients. This notion is supported by experiments conducted in discharges with non-stationary current distributions (produced by current ramping or by retarding the peaking of the current) which indicate a dependence of $\chi_{\text {eff }}$ on the shear [O'Rourke et al., 1991].

\section{c) Heat and particle pulse propagation}

The transport studies described in the previous section concern quasi-steady profiles and result in so-called "power balance" transport coefficients. It has been observed frequently in recent years that transport 


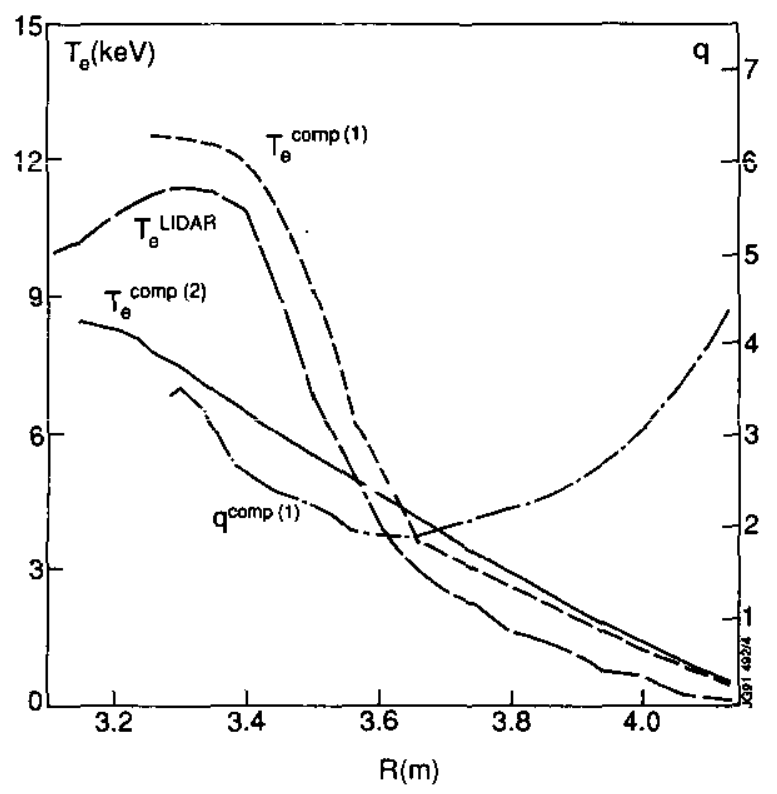

Fig. 10: Simulation of $P E P$-mode indicating that transport must be reduced to the ion neoclassical level $\left(\chi_{t} \sim \chi_{i}=\chi_{\text {ines: }}: \operatorname{comp}(I)\right)$ in the central region with negative shear.

coefficients evaluated from studies of heat and particle pulse propagation i.e. from dynamic processes $\left(\chi^{\text {hp }}\right.$, $\left.\mathrm{D}^{\mathrm{dp}}\right)$ differ from corresponding "power balance" values $\left(\chi^{\mathrm{pb}}\right)$ at given plasma temperature and density. This arises because of the dependence of the transport coefficients not only on $n$ and T, but also on $\nabla n$ and $\nabla T$ (i.e. the relationship between fluxes and gradients can be non-linear). Considerable effort has taken place at JET recently to resolve these apparent contradictions. In this work the linearised, coupled equations for energy and particle transport are used in analysing the heat and particle pulses [Hogeweij et al., 1991]. In studying the propagation of small perturbations it is the "incremental" diffusion coefficients,

which are determined [Lopes Cardozo et al., 1991].

$$
\mathrm{D}^{\mathrm{inc}} \triangleq \mathrm{D}^{\mathrm{eff}}+\frac{\partial \mathrm{D}^{\mathrm{eff}}}{\partial \nabla \mathrm{n}} \nabla \mathrm{n}_{0} \text { and } \chi^{\mathrm{inc}} \equiv \chi^{\text {eff }}+\frac{\partial \chi^{\mathrm{eff}}}{\partial \nabla \mathrm{T}} \nabla \mathrm{T}_{0}
$$

If now, for example, the diffusivities in the plasma depend only on $\nabla T$, we expect (c.f. Fig.11):

$-\chi^{\mathrm{inc}}\left(=\chi^{\mathrm{hp}}\right)$ is larger than the steady state value, $\chi^{\mathrm{eff}}\left(=\chi^{\mathrm{pb}}\right)$

- $D^{\text {inc }}\left(=D^{\mathrm{dp}}\right)$ is similar to the steady state value, $D^{\text {eff }}$

- modelling the pulses with a $\chi^{\text {inc }}$ constant in time implies that $\chi^{\text {eff }}$ varies during the heat pulse.

A detailed analysis of the measurements of heat and particle pulses in JET leads to the following main results [Hogeweij et al., 1991], in agreernent with the above considerations:

- within experimental uncertainties, $\chi^{\mathrm{inc}}$ is the same for ohmic, $\mathrm{L}$ - and H-mode plasmas $\left(\chi^{\mathrm{inc}} \sim 3 \mathrm{~m}^{2} \mathrm{~s}^{-1}\right)$ and is always larger than $\chi^{\mathrm{pb}}$ (by a factor of $\sim 3.2$ for $\mathrm{OH}$ and $\sim 1.6$ for $9 \mathrm{MW}$ of ICRH)

- $\chi^{\mathrm{inc}}$, like $\chi^{\overline{\mathrm{p}} \mathrm{b}}$, depends on the plasma current

- $\chi^{\mathrm{inc}}$ is independent of heating power (determined from a power scan of $3 \mathrm{MA} / 3.1 \mathrm{~T}$ discharges with ICRF heating of between 1 and $9 \mathrm{MW}$ )

- the density pulse diffusion coefficient, $D^{\text {inc }} \sim$ Deff $^{\text {e }} 0.1 \chi^{\text {inc. }}$

The RLW plasma transport model exhibits these features since the diffusivities depend on $\left[\nabla \mathrm{T}_{\mathrm{e}}-\nabla \mathrm{T}_{\mathrm{e}, \mathrm{cr}}\right]$. This | model has successfully simulated heat and particle pulse propagation in JET discharges. Figure 12 [Boucher et al., 1991] shows the comparison with experiment of the simultaneous modelling of temperature and density 


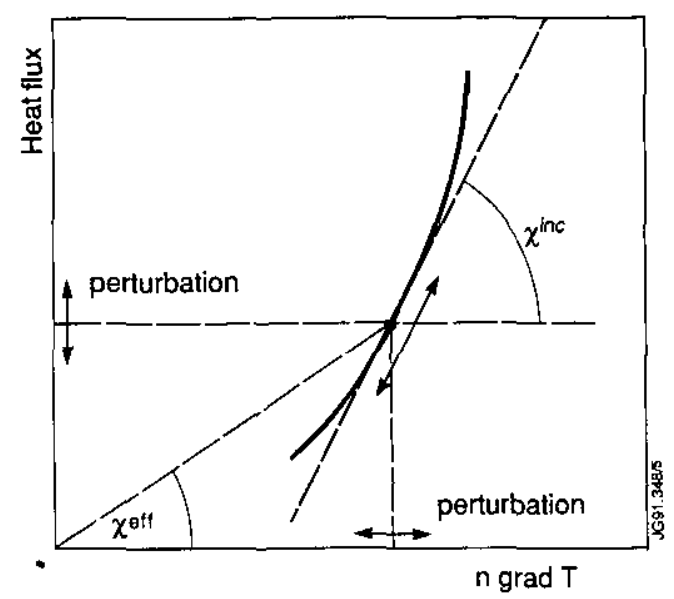

Fig. 11: Sketch to illustrate the difference between the effective diffusivity $\chi^{\text {eff }}$ as determined from a power balance in a stationary plasma and the incremental diffusivity $\chi^{\text {inc }}$ as found from a perturbative experiment. If the heat flux is not proportional to $n \nabla T, \chi^{i n c}=\partial q / \partial(n \nabla T) \neq \chi^{e f}$ and $\chi^{e f}$ varies during the perturbation.

perturbations following the collapse of a sawtooth in a pulse with 7.5 MW of ICRH. The decay of the signal amplitudes with increasing radius and the time traces agree well with experiment for both temperature and density perturbations. The inward propagating density pulse is modelled with an effective particle diffusion coefficient $D_{e, i}^{e f f}$, taken to be proportional to $\chi_{e, i}^{\text {eff }}$ with the required constant of proportionality being $\sim 0.7$. Note that in the simulations the transient depression (dip) observed in the evolution of the density perturbation (indicative of coupling between energy and particle transport) is well represented (Fig. 12).
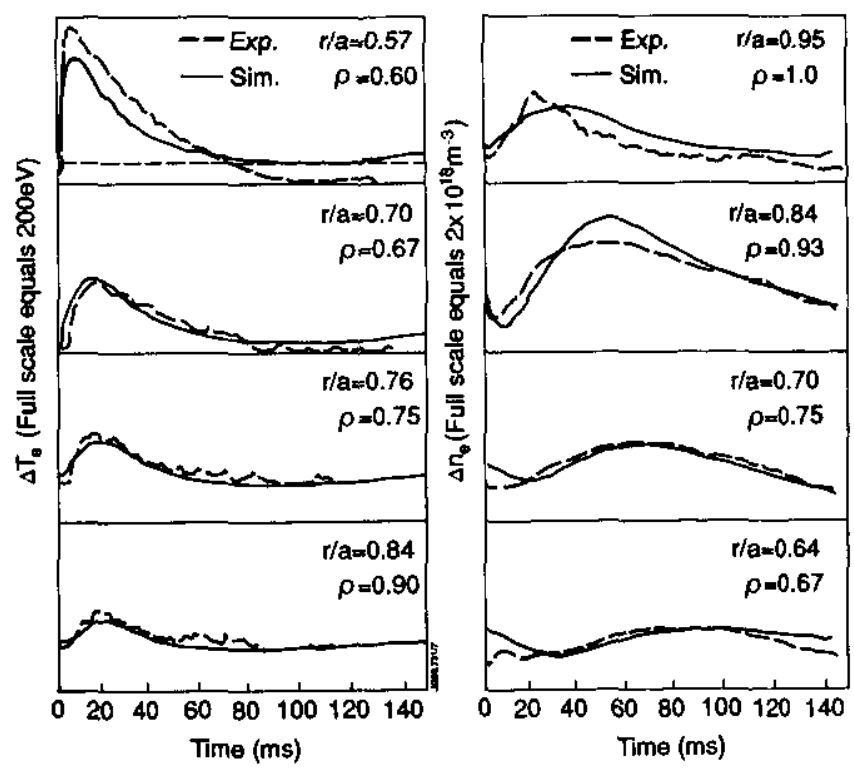

Fig. 12: Comparison with experiment of the simultaneous modelling of temperature and density perturbations following a sawtooth collapse. The agreement is good assuming $D_{e, i}^{c *}=0.7 \chi_{e, i}^{e f f}$ in the simulation. 
In a wider context, it has recently been demonstrated [Sips et al, 1991] that the above analysis using linearised coupled transport equations can also explain the heat and density pulse measurements from TEXT and TFTR. The resulting transport coefficients are very similar for all three experiments even though individual measurements show quite different behaviour of the heat and density pulses. The differences arise primarily from differences in the initial density and temperature perturbations.

\subsection{H-mode Physics and Approach to Steady State}

Here we first discuss the development of two new $\mathrm{H}$-mode scenarios with significance for the application of the $\mathrm{H}$-mode to a NEXT STEP and a reactor: the achievement of $\mathrm{H}$-modes with ICRH, the heating method presently favoured for a NEXT STEP, and the development of ELMy $\mathrm{H}$-mode discharges in which plasma conditions are kept constant for some period of time. In the second part, we describe the influence of the $\nabla B$ drift direction (forward or reversed toroidal field) on $\mathrm{H}$-mode behaviour. This allows a test of theoretical ideas regarding the nature of the $\mathrm{H}$-mode and also provides infomation on how to optimize the distribution of the power load on the inner and outer target tiles.

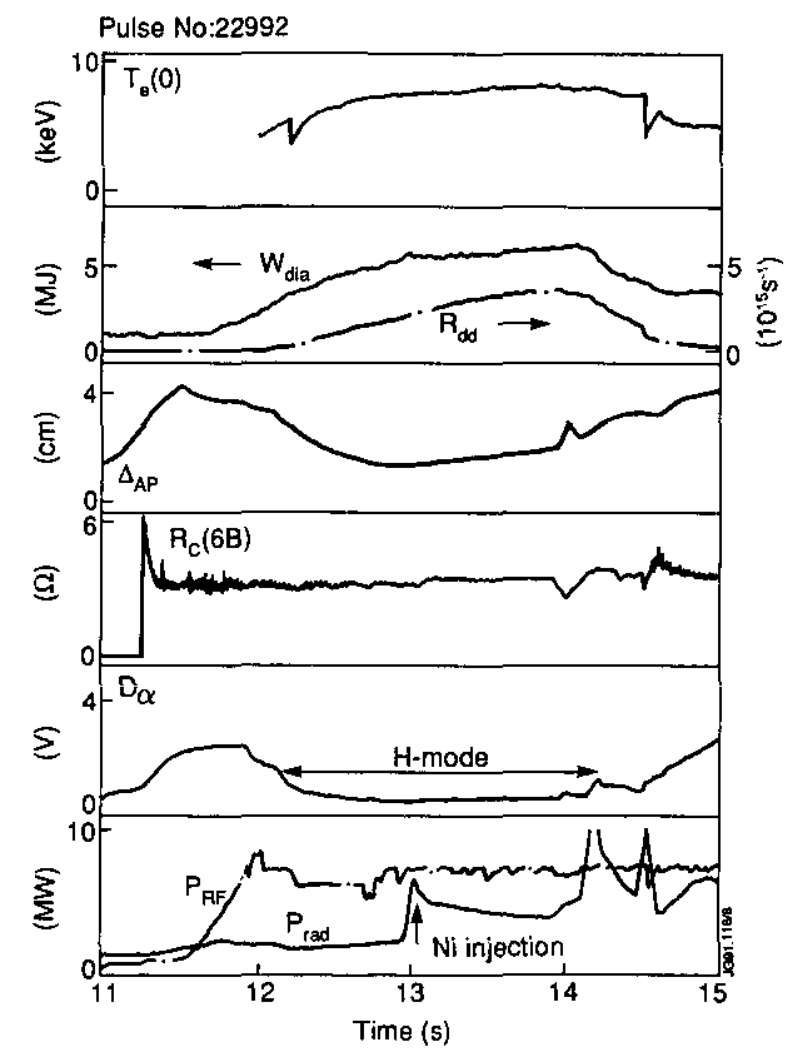

Fig. 13: Various time traces for an ICRF only H-mode (Pulse No: 22992).

\section{a) H-mode scenarios applicable to NEXT STEP/Reactor}

The availability of new machine facilities and evolving skill of the machine operators have led to the development of new H-mode scenarios. Significant progress with ICRF heated H-modes has been achieved following the installation of the beryllium antenna screens and the new ICRF coupling resistance plasmaposition feedback system [Bhatnagar et al., 1991]. With the beryllium antenna screens, the impurity release that can be attributed specifically to ICRH has become negligible, with an estimated contribution to $Z_{\text {eff }}$ of about $0.005 \times \mathrm{P}_{\mathrm{RF}}(\mathrm{MW})$. In addition, the new position control system which uses the RF antenna coupling resistance, via a feedback loop, to control the 
position of the plasma, made it possible to maintain a constant coupling resistance and, as a result, a high heating power (up to $10 \mathrm{MW}$ ) through the $\mathrm{L}$ to $\mathrm{H}$ transition. With these two new features, the operational range of $\mathrm{H}$-modes with ICRF in both dipole or monopole antenna configuration has been considerably extended.

Figure 13 shows an overview of an ICRH-only $\mathrm{H}$-mode in which the new position control system kept the coupling resistance almost constant during the $\mathrm{L}$ to $\mathrm{H}$ and $\mathrm{H}$ to $\mathrm{L}$ transitions. This discharge was a $3 \mathrm{MA} / 2.8 \mathrm{~T}$ DNX deuterium plasma using hydrogen minority heating with the RF antenna in the dipole configuration $\left(k \mid=7 \mathrm{~m}^{-1}\right)$. The power threshold for the $\mathrm{H}$-mode transition in such plasmas is $\sim 5 \mathrm{MW}$, slightly lower than the threshold for similar NBIheated plasmas. The global energy confinement is very similar to that obtained in NBI-heated plasmas with an enhancement factor ranging from 1.8 to 2.8 relative to Goldston L-mode scaling.

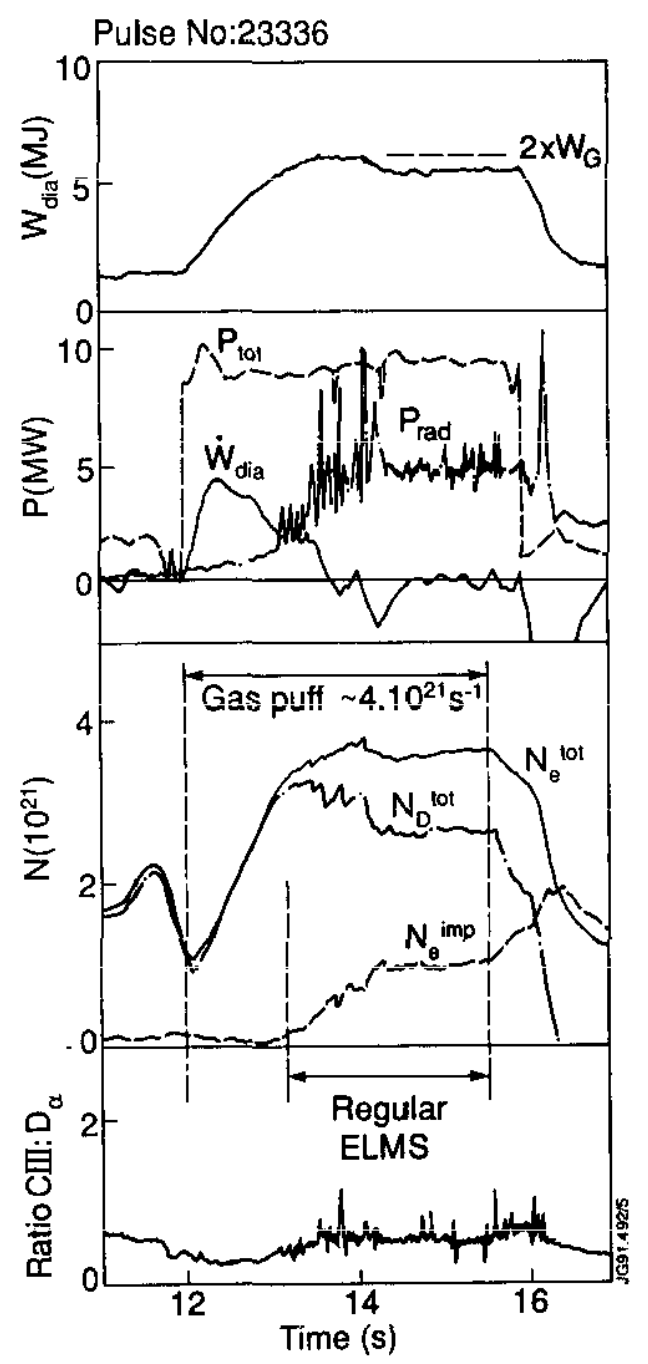

Fig. 14: Various time traces for an ELMy H-mode (Pulse No: 23336) in which plasma parameters were held constant for $2 s$ by the ELMs.

A significant advance made in this campaign, which can be ascribed to the new facilities, was the attainmen of an H-mode with RF antennae in the monopole configuration $(\mathrm{k}||=0)$. However, the power threshold fo conditions similar to those outlined was $\sim 8 \mathrm{MW}$, which is close to the NBI power threshold for thes conditions. In addition, the global energy confinement of these plasmas is lower (by $\sim 30 \%)$ than in the dipol case [Bhatnagar et al., 1991]. 
Whether, in the end, it will be possible to make use of the good confinement properties of the $\mathrm{H}$-mode in future ignition experiments and a reactor will depend on our ability to develop a stationary H-mode plasma. Experiments in DIII-D [Stambaugh et al., 1991] and ASDEX [Wagner et al., 1991] have shown that, under certain conditions, steady-state conditions can be approached in which particle and impurity influxes are controlled by regular ELMs. In JET, ELMs are routinely observed for input powers close to the $\mathrm{H}$-mode threshold power, but disappear as the power is raised. In addition, they are observed in reproducible bursts during hot-ion $\mathrm{H}$-modes.

More regular ELM behaviour has been observed in discharges in which deuterium beams are injected intohydrogen target plasmas [Stork et al., 1991], both at moderate plasma parameters and at high $\beta$. Figure 14 shows such a pulse, in which strong hydrogen gas puffing $\left(140 \mathrm{mbls}^{-1}\right)$ during NBI resulted in frequent ELMs that stabilised the electron and hydrogen particle contents and the plasma energy. The enhancement of energy confinement time dropped somewhat during this period to $\sim 1.8$ times the Goldston L-mode value. On the positive side, however, the high frequency ELMs seem efficient at keeping the carbon content of the plasma stationary even though the outer strike zone remains at $\sim 2700^{\circ} \mathrm{C}$ and is thus a strong source of carbon from radiation - enhanced sublimation, i.e. ELMs exert a strong screening effect on the (light) impurities. Because of lack of experimental time it was not possible to check whether this ELMy H-mode scenario would also work with deuterium as a target and fuelling gas.

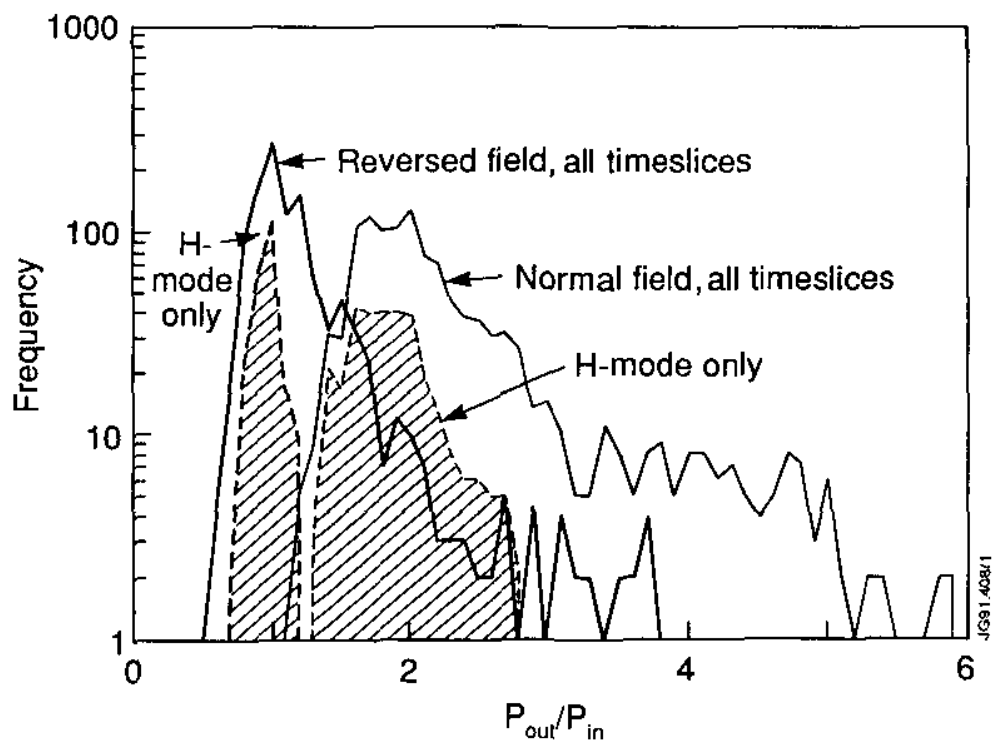

Fig. 15: Frequency distribution of $P_{\text {out }} / P_{\text {in }}$ values from discharges with normal and reversed toroidal magnetic field.

\section{b) Influence of $\nabla B$ drift direction}

Another "experiment" that was carried out to test theoretical ideas that have been put forward to explain the $\mathrm{L}$ to $\mathrm{H}$-transition was to "reverse" the toroidal field in SNX-point discharges ( $\nabla \mathrm{B}$-drift away from X-point). In common with other tokamaks, reversing the toroidal field direction in JET substantially alters the $\mathrm{H}$-mode behaviour [Ward et al., 1991]. The power required to achieve an $\mathrm{H}$-mode is approximately doubled at all values of toroidal field studied (e.g. from 6MW to $12 \mathrm{MW}$ at 2.8T) [Nardone $c$ al., 1991]. Furthermore, the usually observed strong asymmetry in power flow to the target tiles is reduced [Reichle $e t$ al., 1991]. On the other hand, the H-mode energy confinement is found to be very similar with both field directions, as indeed is the L-mode confinement.

Figure 15 shows the frequency distribution of the ratio of powers reaching the outer and inner target tiles, $P_{\text {out }} / P_{\text {in }}$, from many discharges with forward and reversed field, respectively. The powers were measured using an infrared camera looking at one (out of 32 ) band of tiles. The ratio of these powers changes from an average value of $\sim 1.7$ (H-mode) for the $+\nabla \mathrm{B}$-drift direction to $\sim 1$ (H-mode) and $\sim 1.2$ (L-mode) for the $-\nabla B$-drift direction. When 
shadowing of the target tiles is taken into account (correction factor 1.4 ), the corrected ratio between powers to the two strike zones is $\sim 2.4$ in the forward field case and $\sim 0.7$ (H-mode) and $\sim 0.9$ (L-mode) with reversed field. This more symmetric distribution helps to handle large power flows and will therefore have to be considered in designs of NEXT STEP devices.

The change in target power loading with reversal of the toroidal field is accompanied by several changes in measured divertor plasma properties, as indicated in Table II (taken from [Rcichlc et al., 1991]).

Table II. Strike zone on which the larger value of listed plasma property is observed.

\begin{tabular}{l|l|l} 
Plasma Property & Forward Field & Reversed Field \\
\hline Deposited Power & outer & inner (more equal) \\
$\begin{array}{l}\text { Carbon Radiation } \\
\text { Ohmic Heating } \\
\text { Additional Heating }\end{array}$ & $\begin{array}{l}\text { inner } \\
\text { (variable) }\end{array}$ & $\begin{array}{l}\text { outer } \\
\text { (variable) }\end{array}$ \\
Electron Temperature & outer & (more equal) \\
Electron Density & inner & outer
\end{tabular}

These observations can be understood, at least qualitatively, using a scrape-off layer (SOL) model such as that proposed by [Lackner et al., 1984], or the more detailed codes discussed in Sect. 3.3. For fixed midplane SOL density, increasing the power causes an increase in divertor plasma temperature, a decrease in density, and a slight decrease in hydrogen flux to the target. Thus, the more heavily loaded target should display higher plasma temperature, lower density, and lower hydrogen flux. The lower particlc flux reduces carbon sputtering at low target plate temperatures, partially explaining the position of the radiation maximum on the lightly loaded target during the Ohmic phases. For higher power loadings associated with additional heating, target plate temperatures increase to the point where other sputtering processes dominate, and radiation tends to become more equally distributed.

The power flow observations are consistent with a description which separates the flow of heat across the separatrix into two components [Hinton, 1985]:

1) The underlying transport, assumed to carry heat predominantly through the major radius side of the torus (independent of the direction of the field), and

2) an additional flow near the $X$-point, flowing into one side of the plasma and out of the other, which reverses when the field is reversed. In cylindrical geometry this would not contribute to the total power crossing the separatrix.

The measurements (ratios of approximately 3 in forward and 1 in reversed field) are consistent with an underlying heat flow to the outer and innertargets of $5 / 8 \mathrm{P}_{\text {tot }}$ and $3 / 8 \mathrm{P}_{\text {tot }}$, respectively (where $\mathrm{P}_{\text {tot }}$ is the total powercarried across the separatrix). The additional flow would then be $1 / 8 \mathrm{P}_{\text {tot }}$, flowing from the small to large major radius side of the $\mathrm{X}$-point with forward toroidal field, reversing when the field reverses. In toroidal geometry, the additional flow may produce a net flow of heat across the separatrix. This should be only $\sim \varepsilon \times 1 / 8 \mathrm{P}_{\text {tot }}$ ( $\varepsilon$ the inverse aspect ratio) and is rather small. It is hard to see how this slight change in confinement could be responsible for the doubling of the $\mathrm{H}$-mode threshold power in reversed field discharges.

\subsection{Divertor Physics and Impurity Control}

Numerical and analytical modelling carried out during the past year have led to advances in our understanding of the factors which affect the performance of an open divertor. The work has had two majorgoals. The first is to predict the performance of the pumped divertor planned for installation in JET in 1992, and the second is to model present "divertor" (i.e. X-point) operation in JET, in order to assess the SOL/divertor models. Because the parameters under which the pumped divertor is expected to operate efficiently will differ markedly from those found in the limited number of X-point discharges analysed so far, the dominant physical phenomena governing divertor performance differ between the two cases. 


\section{a) Modelling of the pumped divertor}

In order to ensure effective operation at high SOL power, the pumped divertor phase will be characterized by high densities in the SOL. In addition, the distance $\Delta \mathrm{X}$ between the target plates and the $\mathrm{X}$-point is relatively large, varying from roughly $20 \mathrm{~cm}$ to $40 \mathrm{~cm}$, depending on the equilibrium chosen. This combination of factors implies that the divertor plasma will be quite opaque to both hydrogen and impurity neutrals emanating from the plate, so that sources of hydrogen and impurity ions will be localized in the divertor plasma near the targets, well away from the $\mathrm{X}$-point and main plasma. This is a necessary but not sufficient condition for retention of target-produced impurities in the divertor region Retention also depends on a balance between several forces acting on the impurity ions, the most important are the hydrogenic ion-impurity friction force directed towards the target, and the ion temperature gradient force, directed away; in steady state the impurity concentration adjusts to balance the difference between the two forces through its own pressure gradient, $\mathrm{dp}_{\mathrm{z}} / \mathrm{ds}$, where $\mathrm{s}$ is distance along the field line.

Analytical and numerical work describing this mode of operation is reported in [Keilhacker, Simonini et al., 1991, Keilhacker et al., 1991, and Vlases and Simonini, 1991]. At high values of mid-plane separatrix density $n_{b}$, for a given value of power $P_{i}$ flowing through the ion channel in the SOL, the divertor density $n_{d}$ and hydrogen flux to the target $\Gamma_{\mathrm{d}}$ are large, and the divertor flow established by local recycling alone is sufficient to retain the impurities. The simplified analytical model shows that this corresponds to achieving a divertor ion temperature $T_{\mathrm{di}}$ below approximately $20 \mathrm{eV}$, nearly independent of $\mathrm{P}_{\mathrm{i}}$. Since $\mathrm{T}_{\mathrm{di}}$ is a function of $n_{\mathrm{b}}$ and $\mathrm{P}_{\mathrm{i}}$, an expression can be written for the minimum value of $n_{b}$ at which retention is achieved as a function of power:

$$
\mathrm{n}_{\mathrm{b}, \min }=\frac{q_{i}}{\left((7 / 2) q_{i} L / K_{o i}\right)^{2 / 7} C_{o} T_{d}^{1 / 2}}
$$

i.e., $\mathrm{n}_{\mathrm{b}, \mathrm{min}}$ scales approximately as $\mathrm{q}_{\mathrm{i}}^{5 /}$. Here $\mathrm{q}_{\mathrm{i}}$ is $\mathrm{P}_{\mathrm{i}}$ divided by $\mathrm{SOL}$ area, $\mathrm{L}$ is the distance along the field line from target to stagnation point and $\mathrm{K}_{\mathrm{oi}}$ and $\mathrm{C}_{\mathrm{o}}$ are constants. Figure 16 [Keilhacker et al., 1991] shows that midplane separatrix densities of $\sim 10^{20} \mathrm{~m}^{-3}$ will be required for $\mathrm{P}_{\mathrm{i}}>10 \mathrm{MW}$.

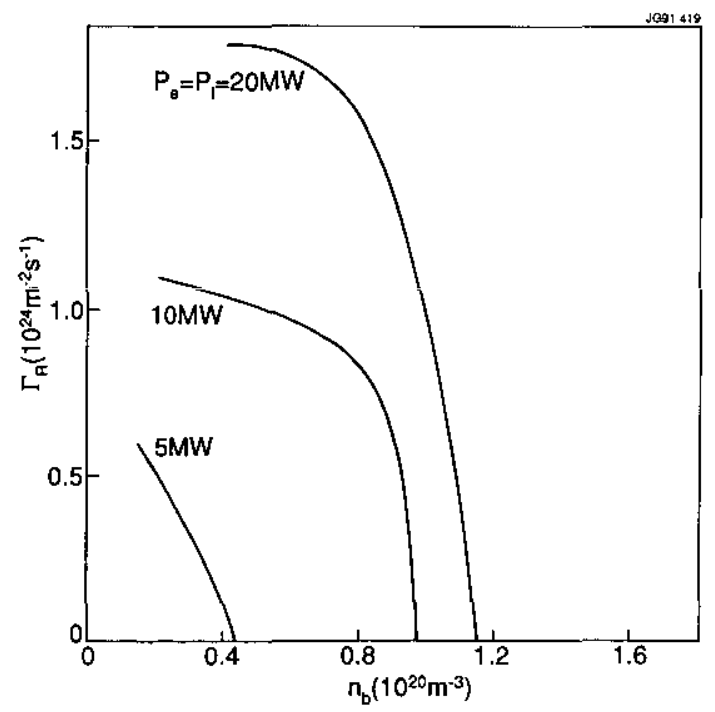

Fig. 16: Recirculated hydrogen flow required for $90 \%$ retention of impurities in the divertor region versus midplane separatrix density for three values of ion power flow in the scrape-off layer.

As $n_{b}$ drops below $n_{b}$,min, the friction forces arising from the local recycling are insufficient to overcome the ion temperature gradient force, which extends along the entire field line to the stagnation point, and an additional flow must be induced in the SOL, either by ducting ("internal recirculation") or by direct particle injection ("extemal recirculation") with compensating pumping from the divertor region to maintain steady state. As described in [Keilhacker et al., 1991], the required flow depends principally on $\mathrm{P}_{\mathrm{i}}$, and becomes relatively independent of $\mathrm{n}_{\mathrm{b}}$ for densities well below $n_{b}, \min$ (Fig. 16). For the reference case of injection of all of the recirculation flow at the X-point, the required flow scales as $P_{i}^{5 / 7}$, with weaker dependence on the connection length and $n_{b}$. 


\section{b) Divertor assessment for the 1990 campaign}

Analysis of divertor performance has begun for a limited number of representative X-point discharges from the 1990 campaign. The parameters for these discharges differ from those discussed above for high power operation of the pumped divertor in three principal ways. First, the target - $\mathrm{X}$-point separation, $\Delta \mathrm{X}$, is much smaller, varying from 5 to $15 \mathrm{~cm}$, although the connection lengths along the field line between target and X-point are comparable to those in the pumped divertor. Second, the values of $\mathrm{n}_{\mathrm{b}}$ are lower, in the range of $1-2 \times 10^{19} \mathrm{~m}^{-3}$. Finally, the sum of the ion and electron power flows into the divertor plasma is low, typically on the order of 2-3 MW. These factors result in a very much lower divertor density, $\mathrm{n}_{\mathrm{d}}$, than discussed above for the pumped divertor phase. Consequently, the $\mathrm{X}$-point divertor plasma for the analysed 1990 discharges is not fully opaque to hydrogen and impurity neutrals. The neutral hydrogen can travel long distances from the target ("distant recycling") and reenter the SOL well upstrearm. This increases the flow in a large portion of the SOL, producing the beneficial effects of increasing the ion friction force and reducing the temperature gradient, since much of the ion energy is carried by convection. Thus, the calculations indicate that any impurity ions bom in the divertor plasma are well retained. However, at the low

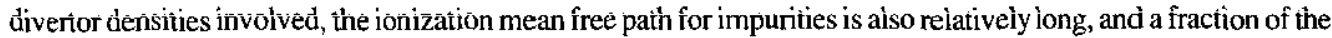
neutral impurity atoms sputtered from the target plate can go directly into the main plasma. In other words, at the low end of the density range for these shots, the screening effect of the divertor is essentially lost.
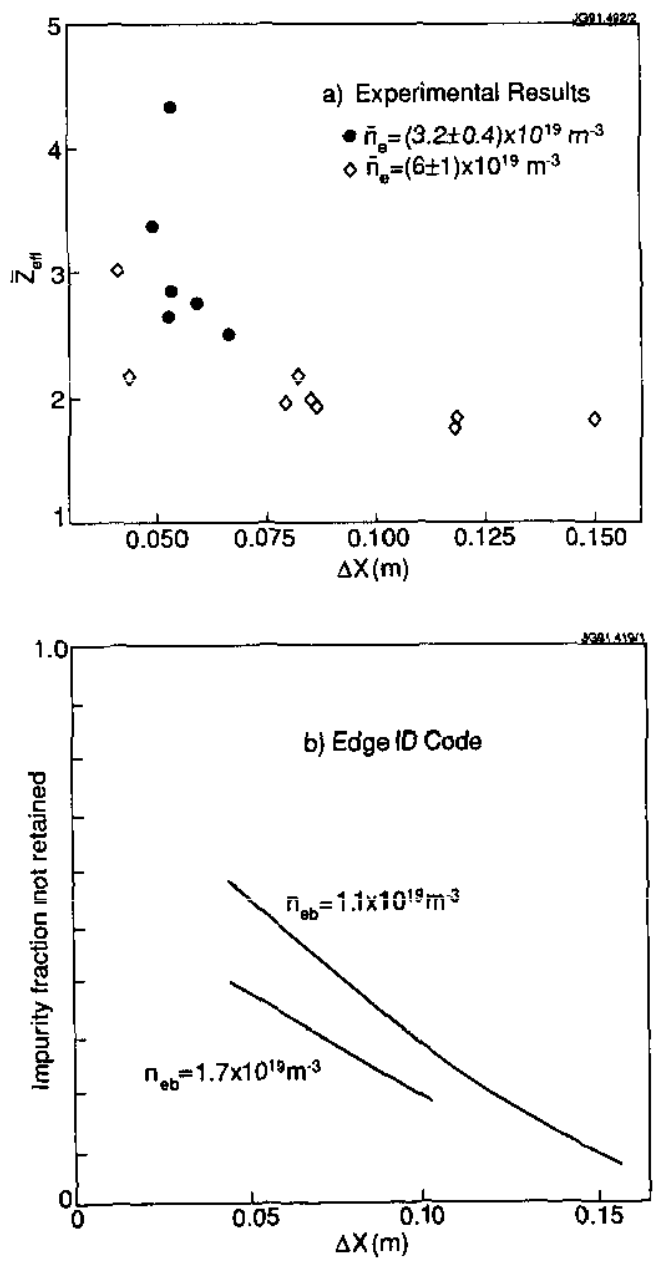

Fig. 17: (a) Variation of $\bar{Z}_{\text {ef }}$ in $H$-mode with distance between upper X-point and target tiles $(C)$ for two different densities $\left(I_{p}=3.1 \mathrm{MA}, P_{N B t}=95 \mathrm{MW}\right)$, and $(b)$ comparison with code results. 
As indicated above, we require $\Delta \mathrm{X} \gg>\lambda_{\mathrm{i}, \mathrm{z}}$, the ionization mean free path for impurities, which scales as $1 / n_{d}$, as a necessary condition for impurity retention. One thus expects a strong dependence of retention on both $\Delta X$ and $n_{d}$ (which is determined by $n_{b}$ and $P_{j}$ ). This is corroborated by the code results of Fig. 17b, which show the fraction of all divertor/SOL impurities which reside in that portion of the $\mathrm{SOL}$ which is adjacent to the main (closed flux surface) plasma, for a set of runs at a fixed input power of $P_{e}=P_{i}=1 \mathrm{MW}$, At a midplane separatrix density of $1 \times 10^{19} \mathrm{~m}^{-3}$, there is a strong dependence of retention on $\Delta \mathrm{X}$ due to the penetration of neutral impurities directly into the main plasma. At $n_{b}=1.7 \times 10^{19} \mathrm{~m}^{-3}$, there is better retention over the range of $\Delta X$ from 5 to $10 \mathrm{~cm}$, since the impurity sources are now more localized to the divertor region, and the friction force exceeds the ion thermal force.

A quantitative comparison between modelling and experiment for the 1990 campaign is not possible due to insufficent diagnostic information. However, experimental confirmation of the trends described above is shown in Fig. 17a, from [Morgan et al., 1991]. Here we use $Z_{\mathrm{eff}}$ as an indicator of divertor retention. At a value of line averaged electron density $\tilde{n}_{e} \sim 3 \times 10^{19} \mathrm{~m}^{-3}$, corresponding approximately to $\mathrm{n}_{\mathrm{b}}=1 \times 10^{19} \mathrm{~m}^{-3}$, we see a strong dependence of $Z_{\mathrm{eff}}$ on $\Delta \mathrm{X}$, while at double the line-averaged density, the dependence is very weak, indicating good retention until the $\mathrm{X}$-point is nearly at the target.

\subsection{Experimental Simulation of Alpha-Particle Effects}

By virtue of the plasma conditions it $c$ an produce, JET is in an unique position to simulate many single and collective energetic particle effects which will be encountered under reactor conditions. During the 1990 campaign, continued progress was made on simulation of such alpha-particle effects, and in addition a preliminary assessment of helium ash removal was made.

\section{a) Single particle effects: slowing down and diffusion}

The study of the burnup of tritons released from $\mathrm{d}$-d reactions is of special interest because the $1.0 \mathrm{MeV}$ tritons possess similar kinematic properties to the $3.5 \mathrm{MeV}$ alpha-particles released from d-t reactions. The triton birth profile is given by the emission profile of the associated $2.5 \mathrm{MeV}$ neutrons, and their radial movement whilst slowing down is indicated by the emission of $14 \mathrm{MeV}$ neutrons from their burnup (t-d) reactions.

Previous work, in which the time-dependence of the total $14 \mathrm{MeV}$ neutron production was related to the total 2.5 $\mathrm{MeV}$ neutron production, has indicated that the confinement and slowing down of the fast tritons is in good accord with classical expectations, provided the slowing down time was less than $0.5 \mathrm{~s}$, as is the case for the majority of JET discharges [Conroy et al., 1988]. During the 1990 experimental campaign, the bumup studies were extended to include measurements of the radial profile of the $14 \mathrm{MeV}$ neutrons from t-d reactions [Jarvis et al., 1991]. The radial profiles of both 2.5 and $14 \mathrm{MeV}$ neutron emission were assumed to have the simple form $\left(1-\mathrm{r}^{2} / \mathrm{a}^{2}\right)^{\alpha}$. The resulting peaking factors, $\alpha$, for a particular discharge (Pulse No: 22517) with $12 \mathrm{MW}$ of beam heating are shown in Fig. 18. The triton slowing-down time for the conditions of this discharge was about 1.4s, showing that the abrupt peaking of the $14 \mathrm{MeV}$ profile at $16.8 \mathrm{~s}$ corresponds with the peaking of the $2.5 \mathrm{MeV}$ profile that takes place at $15.5 \mathrm{~s}$ when the beam heating is suddenly reduced. The peaking factor for $14 \mathrm{MeV}$ neutrons produced by tritons generated during the main heating period ( 14 to $15.5 \mathrm{~s}$ ) is 4.5 and is to be compared with the $2.5 \mathrm{MeV}$ peaking factor of 5.8 . This slightly greater width for the $14 \mathrm{MeV}$ neutron profile is expected as a consequence of the finite Larmor radius of the tritons. It is worth noting that it has not so far been possible to identify unambiguously any effects on the triton burnup that are attributable to sawtooth crashes.

An important observation that can be drawn from Fig. 18 is that there is no evidence for triton diffusion; numerical studies indicate that the constancy of $\alpha$ from 15.5 to $16.5 \mathrm{~s}$ demonstrates that the triton diffusion coefticient must be very small $\left(<<0.1 \mathrm{~m}^{2} / \mathrm{s}\right)$. Consequently, charge exchange losses are now believed to be the reason for the unexpectedly low triton bumup observed in discharges with a long slowing down time ( $\geq 2 \mathrm{~s})$ [Conroy et al., 1990].

\section{b) Collective effects}

The ICRF heating of light minority ions provides an opportunity to simulate collective effects of fast particle populations in the plasma. With both hydrogen and ${ }^{3} \mathrm{He}$ minority heating schemes, fast particle energy contents of 


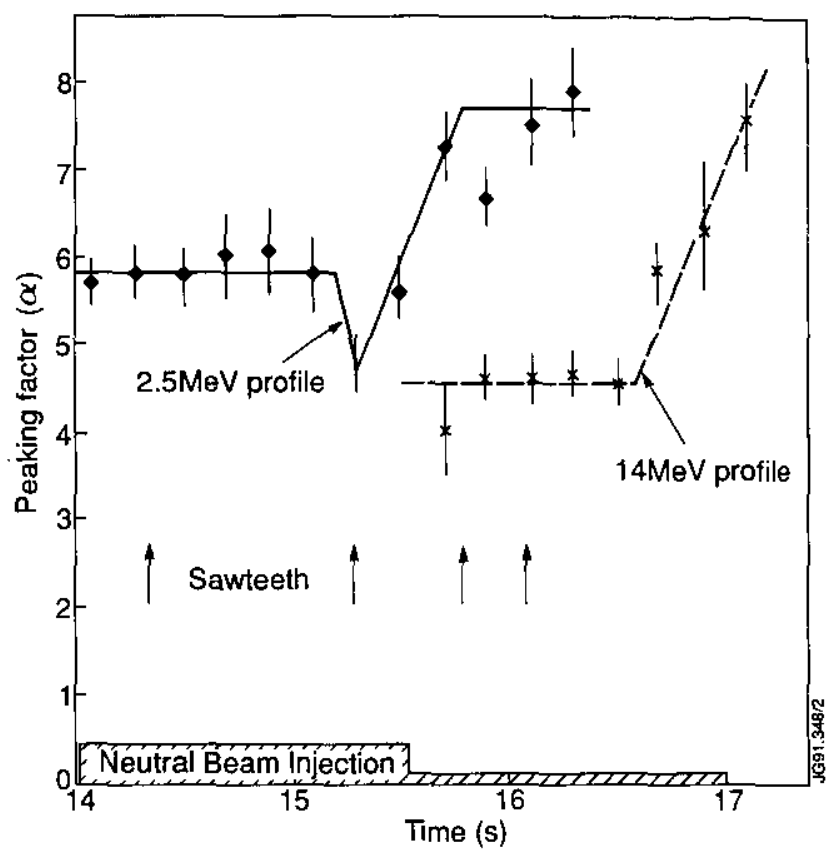

Fig. 18: Profile peaking factors for both 2.5 and $14 \mathrm{MeV}$ neutrons as obtained with the neutron profile monitor for a discharge with 12MW NBI heating (Pulse No: 22517).

Times at which sawtooth crashes occur are indicated.

$>3 \mathrm{MJ}$ have been achieved. The $3 \mathrm{He}$ heating schene is of particular interest since the $3 \mathrm{He}-\mathrm{D}$ fusion reactions can be studied directly by exploiting the ${ }^{3} \mathrm{He}+\mathrm{D} \rightarrow{ }^{5} \mathrm{Li}+\gamma$ reaction, in which the $\gamma$-rays carry off energy of $\sim 16.6 \mathrm{MeV}$.

As described [Jacquinot and Sadler, 1991], there have been recent advances in understanding of the efficiency of the RF heating process. There are two effects to be considered: saturation of the central electron temperature with increasing applied RF heating power (see Sect. 2.1) and failure of the fusion reaction power to achieve the predicted levels. These difficulties are resolved when the observed quantities $\left(\mathrm{T}_{\mathrm{e}}\right.$ and fusion power $)$ are compared with the central heat deposition by the fast particles (calculated without over-simplification and taking fast particle orbit effects into account). The central electron temperature is now found to increase approximately linearly with the heating power deposited within the half-width of the electron temperature profile [Cordey et al., 1991]. The fusion reaction power achieved in a particular discharge depends sensitively on the minority ion concentration (which is not well known). However, as has been shown [Jacquinot and Sadler, 1991], the maximum fusion yield obtainable at optimum minority ion concentration should scale linearly with perpendicular energy content of the fast ions:

$$
P_{\text {fus }}[\mathrm{MW}]=0.12\left(\mathrm{n}_{\mathrm{d}} / \mathrm{n}_{\mathrm{e}}\right) \mathrm{W}_{\text {fast }}[\mathrm{MJ}] \text {, }
$$

provided the tail temperature exceeds the critical energy, typically about $300 \mathrm{keV}$.

Figure 19, taken from [Sadler et al., 1991], shows the measured fusion power plotted against the fast ion energy content along with curves of the variation of the theoretical maximum for two assumed fuel concentrations. The figure indicates that the theoretical maximum has been approached only for a few clean plasma discharges (unfortunately, $n_{d} / n_{e}$ concentrations of about 0.5 were normally observed in these experiments). It is interesting to note that the fusion power in the ${ }^{3} \mathrm{He}-\mathrm{D}$ reaction is almost exclusively released in the form of charged particle kinetic energy and that the record value of about $140 \mathrm{~kW}$ contribution to plasma heating is equivalent to that which would be produced by $0.7 \mathrm{MW}$ of total fusion power from D-T reactions.

Within the limitations of the existing diagnostics, no deleterious consequences of collective fast ion behaviour have been observed, despite the highly anisotropic velocity distribution function and fast ion toroidal $\beta$ of up to $8 \%$. 


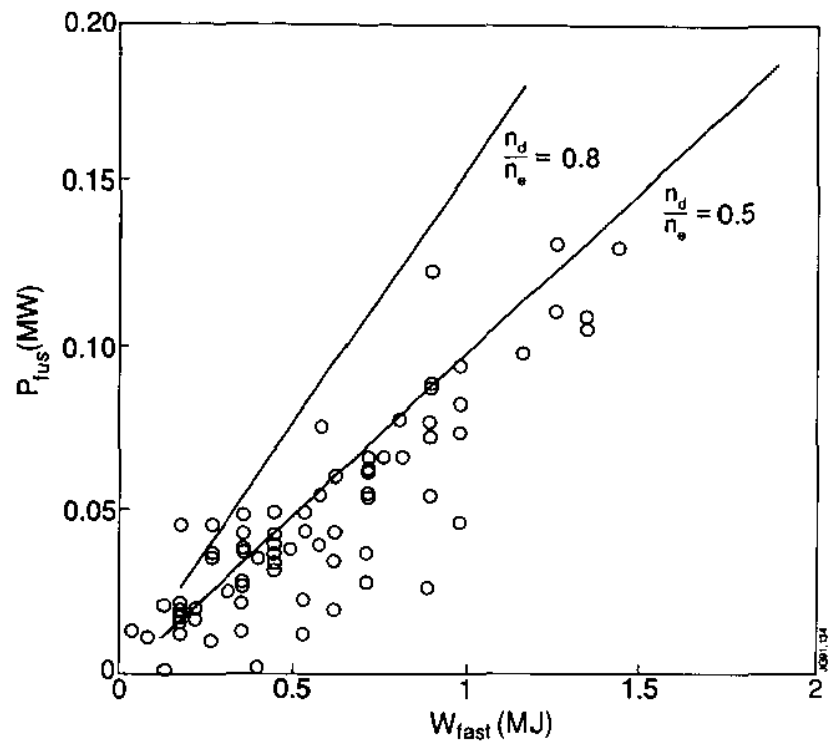

Fig. 19: Fusion power generated as a function of fast ion energy content (experimental points). The theoretical maximum achievable powers for $n_{\mathrm{d}} n_{\mathrm{e}}=0.5$ and 0.8 are also shown (solid lines).

Fishbones are frequently excited under appropriate conditions, but there is no evidence of any significant reduction in the fast ion content of the plasma, other than that which occurs following a sawtooth crash.

\section{c) Helium ash transport}

In order to achieve a steady-state burning plasma such that the alpha-particle production rate is balanced by the $\mathrm{He}$ removal rate, one can show that the condition $\tau_{\mathrm{p}}(\mathrm{He}) / \tau_{\mathrm{E}} \leq 10 \varepsilon$ must be satisfied, where $\varepsilon$ is the pumped fraction for helium (e.g. [Reiter et al., 1990]). For this reason knowledge of the relationship between particle and heat transport is of fundamental importance.

In JET He ash production and transport were studied by the simultaneous use of He beam injection, providing a centrally peaked and well defined source of $\mathrm{He}^{2+}$ ions, and of $\mathrm{D}$ diagnostic beams for an absolute measurement of the temporal and spatial evolution of the $\mathrm{He}^{2+}$ density profile by charge exchange spectroscopy (CXS). In the experiments [Jones et al., 1991 ] a short $(\sim 0.5 \mathrm{~s}) \mathrm{He}^{\circ} \mathrm{NBI}$ pulse ( $\left.\sim 3-6 \mathrm{MW}\right)$ was applied to a low to moderate density deuterium plasma $\left(\left\langle\mathrm{n}_{\mathrm{e}}\right\rangle \sim 1-3 \times 10^{19} \mathrm{~m}^{-3}\right)$, sufficient to produce an average minority concentration $\mathrm{n}_{\mathrm{He}} / \mathrm{n}_{\mathrm{e}} \sim 10 \%$. The time evolution of $n_{\mathrm{He}}(0)$ was measured via a central vertical $\mathrm{CX}$ viewing channel whose sensitivity had been absolutely calibrated with an accuracy of $30 \%$. In addition, radial profile measurements $n_{\mathrm{He}}(\mathrm{r})$ were available from a horizontal multichord array within the region $\mathrm{r} / \mathrm{a} \leq 0.5$.

Figure 20 compares the evolution of the partial profile of $n_{\mathrm{He}}$ for $\mathrm{r} / \mathrm{a} \leq 0.5$ with that of the time integrated $\mathrm{He}^{\circ} \mathrm{NBI}$ deposition profilel $\int_{0}^{t} S_{\text {Hie }}(r) d t$ (calculated using the PENCIL code) for a sawtoothing $\mathrm{H}$-mode discharge. The times indicated are relative to the start of the $\mathrm{He}^{\circ} \mathrm{NBI}$ pulse. The rate of accumulation of $\mathrm{He}^{2+}$ is much less than would be expected, if it were assumed simply that the plasma core fills with $\mathrm{He}^{2+}$ ions, as given by the $\mathrm{He}^{2+} \mathrm{NBI}$ source, with no $\mathrm{He}^{2+}$ outflux. This implies that a strong $\mathrm{He}^{2+}$ outflux $\Gamma_{\mathrm{He}}$ must in fact be established.

These and similar profiles have been used to determine the effective He particle diffusion coefficients given by $\mathrm{D}_{\mathrm{He}}=-\Gamma_{\mathrm{He}} / \mathrm{n}_{\mathrm{He}}$ and the core particle confinement times (within $\mathrm{r} / \mathrm{a}=0.5$ ) are defined by:

$$
\tau_{H e}(0.5)=\int_{0}^{0.5} n_{H i e} J_{v} d X /\left[\int_{0}^{0.5}\left(S_{N B}^{H e}-\dot{n}_{H e}\right) J_{v} d X\right]
$$




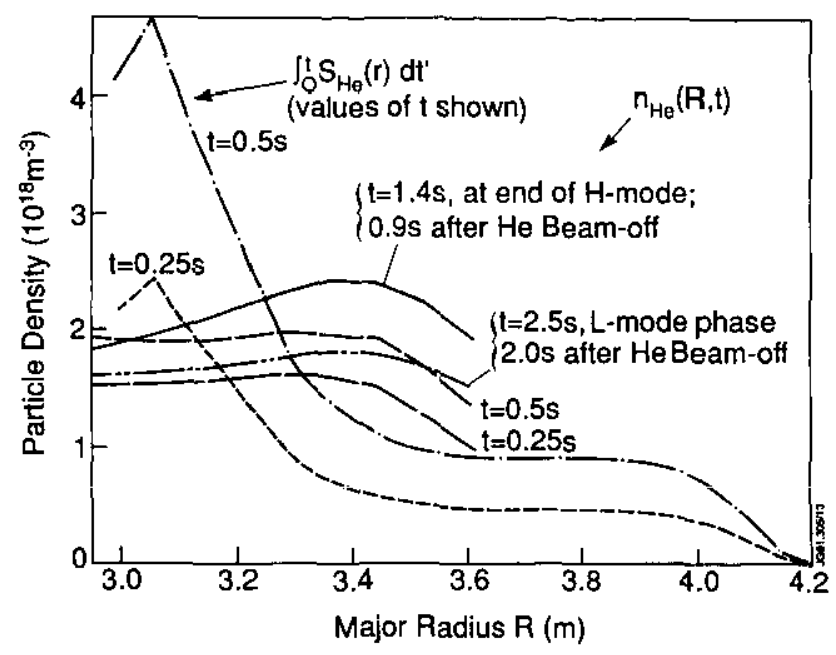

Fig. 20: $\mathrm{He}^{2+}$ density profiles and integrated He NB deposition profiles for a sawtoothing $H$-mode discharge (Pulse No: 23198 with $P_{t o t}=7 M W$ ). The times indicated are relative to the start of the $\mathrm{H} e^{\circ} \mathrm{NBI}$ pulse.

where $\mathrm{J}_{\mathrm{v}} \mathrm{dX}$ is the volume element. The latter are then compared with analogously defined core energy confinement times $\tau_{\mathrm{E}}(0.5)$.

Tentatively, the results can be summarised as follows [Jones $\mathrm{et}$ al., 1991]:

- He transport in the plasma core $(\mathrm{r} / \mathrm{a} \leq 0.5)$ is similar in $\mathrm{L}$ - and H-mode discharges (sawtoothing and nonsawtoothing ones) with $\mathrm{D}_{\mathrm{He}} \sim 0.5 \mathrm{~m}^{2} \mathrm{~s}^{-1}$ at $\mathrm{r} / \mathrm{a}=0.5$ and is sufficient to effectively remove $\mathrm{He}^{2+}$ from the plasma core.

- The ratio of core confinement times $\tau_{\mathrm{Hc}}(0.5) / \tau_{\mathrm{E}}(0.5)$ was $\leq 1.5$ for a sawtoothing, low power $(7 \mathrm{MW}) \mathrm{NBI} H-$ mode and $\leq 3$ for a sawtooth-free, combined heating $(12 \mathrm{MW}) \mathrm{H}$-mode. This is a favourable result since to avoid ash accumulation in the plasma core it should suffice to meet the condition $\tau_{\mathrm{He}} / \tau_{\mathrm{E}} \leq 10$ there.

\section{SUMMARY AND CONCLUSIONS}

In the preceding sections of this paper we have first reported on improvements in the performance of JET plasmas during the last campaign, and then discussed progress in understanding selected physics aspects of the diverse operating modes obtainable in JET.

In separate discharges, $\mathrm{JET}$ has achieved $\mathrm{n}_{\mathrm{e}}(0) \sim 4 \times 10^{20} \mathrm{~m}^{-3}, \mathrm{~T}_{\mathrm{j}}(0) \sim 30 \mathrm{keV}, \mathrm{T}_{\mathrm{e}}(0) \sim 12 \mathrm{keV}, \tau_{\mathrm{E}} \sim 1.8 \mathrm{~s}$, and $\beta \sim 5.5 \%$. Thus, the parameters required of a reactor plasma have all been achieved individually. In certain pulses, JET has achieved $\mathrm{n}_{\mathrm{D}}(0) \tau_{\mathrm{E}} \mathrm{T}_{\mathrm{i}}(0)=8-9 \times 10^{20} \mathrm{~m}^{-3} \mathrm{~s} \mathrm{keV}$ with $\mathrm{T}_{\mathrm{i}}(0)=10-30 \mathrm{keV}$. This is close to the plasma conditions needed for $Q_{D T}=1$, and within a factor of six of that required for an ignited reactor plasma. These values, however, were only achieved in a transient state, which in most cases was terminated by a rapid influx of impurities caused by excessive heating of the non-optimized target plates.

Several features have emerged from our transport studies during the past year. First, a link between anomalous particle and energy transport seems to be firmly established, with simulations indicating that $D^{\text {eff }} \sim 0.2-0.5$

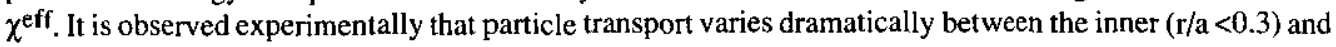
outer $(\mathrm{r} / \mathrm{a}>0.3)$ plasma regions. In the inner region, particle transport is close to the neoclassical level in many cases, with $D_{\text {imp }} \sim 0.1 \mathrm{~m}^{2} \mathrm{~s}^{-1}$ for $\mathrm{OH}, \mathrm{L}$ and $\mathrm{H}$ modes and $\mathrm{D}_{\mathrm{e}} \sim 0.03 \mathrm{~m}^{2} \mathrm{~s}^{-1}$ after pellet injection, while $\chi^{\text {eff }}$ is about $0.5 \mathrm{~m}^{2} \mathrm{~s}^{-1}$ and $0.3 \mathrm{~m}^{2} \mathrm{~s}^{-1}$ respectively for $\mathrm{L}$ and $\mathrm{H}$ modes. In the outer region, $\mathrm{D}_{\mathrm{imp}} \sim 3-5 \mathrm{~m}^{2} \mathrm{~s}^{-1}$ in the $\mathrm{L}$ mode, which is about 100 times neoclassical, while in the $\mathrm{H}$ mode it is a factor of 10 less. Outer region values 
of $\chi^{\text {eff }}$ are $5 \mathrm{~m}^{2} \mathrm{~s}^{-1}$ and $2 \mathrm{~m}^{2} \mathrm{~s}^{-1}$ respectively for $\mathrm{L}$ and $\mathrm{H}$ modes. There is evidence that modifying the shear changes the confinement properties of the plasma, and in particular that regions of reversed shear are correlated with low energy transport, in some cases comparable to neoclassical values for the ions.

Most "NEXT STEP" and reactor designs utilize some form of H-mode scaling. In order to achieve steady state Hmodes, by preventing accumulation of $\mathrm{He}$ ash and other impurities, it is thought that either sawteeth in the core and/ or ELMs at the edge will be necessary. JET has achieved ELMy H-modes under certain operating conditions during the past campaign while maintaining an energy confinement time a factor of 1.8 higher than Goldston L-mode scaling. Furthermore, experimental simulation of He ash transport indicates effective removal of alpha-particles from both $\mathrm{L}$ and $\mathrm{H}$ mode plasma with $\tau_{\mathrm{He}}(\mathrm{core}) / \tau_{\mathrm{E}}(\mathrm{core}) \leq 3$.

Significant effort has gone intodivertor modelling at JET over the past years. This modelling, based on 1 1/2Dcodes and analytic analysis, has shown that in the pumped divertor phase of JET, retention of target-produced impurities can be expected, at a given power level, if the midplane separatrix density is high enough that the divertor ion temperature is maintained below about $20 \mathrm{eV}$; the required density scales as the SOL ion power flow to the $5 / 7$ power. For lower separatrix densities, impurity retention requires that the flow arising near the target plates from local recycling be supplemented by an additional "recirculation" flow introduced beyond the local recycling zone. Preliminary application of these models to the 1990 campaign shows qualitative agreement with experiment, although the dominant effects expected in high power pumped divertor operation are somewhat different from those encountered in the 1990 shots analyzed to date.

The final example of improved physics understanding dealt with alpha-particle effects, which represent a relatively untested area of reactor plasma physics. It has been shown that the slowing down of tritons is classical, and that the effective diffusion coefficient during this process is very small $\left(\mathrm{D} \ll 0.1 \mathrm{~m}^{2} \mathrm{~s}^{-1}\right)$. In addition, our investigation of ICRF heating of light minority ions $\left(\mathrm{H},{ }^{3} \mathrm{He}\right)$ has produced values of $\beta_{\text {fast }}$ up to $8 \%$ with no evidence of deleterious effects due to collective fast ion behaviour. These results suggest that the prospects for effective alpha-particle heating in next step devices and reactors appear good.

In conclusion, during the 1990 campaign, $J \mathrm{ET}$ continued to make steady progress towards the realization of fusion power. Technical advances and the development of new operating scenarios led to improved plasma performance in several areas. In addition, specific studies served to clarify a number of physics issues confronting successful design and operation of NEXT STEP devices.

\section{ACKNOWLEDGEMENTS}

I would like to thank many of my colleagues for their contributions and for clarifying discussions, in particular J Cordey, N Jarvis, T T C Jones, J O'Rourke, A Sips, A Taroni, F Tibone, G Vlases, D Ward and M Watkins.

\section{REFERENCES}

Balet, B. et al. (1990). Nucl. Fusion 30, 2029.

Behringer, K. et al. (1988).Proc.15thEur. Conf. on Contr.FusionandPlasmaHeating, Dubrovnik, 1988, Vol. 12B, Part I, p. 338

Bhatnagar, V.P. et al. (1991). Plasma Phys. and Contr. Fusion 33,99.

Bhatnagar, V.P. et al. (1991). Proc. 18th Eur. Conf. on Contr. Fusion and Plasma Phys., Berlin, 1991, Vol. 15C, Part I, p. 369.

Boucher, D. et al. (1991). Proc. I8th Eur. Conf. on Contr. Fusion and Plasma Phys., Berlin, I991, Vol. 15C, Part I, p. 177.

Bussac, M.N. et al. (1975). Phys. Rev. Lett. 35, 1638.

Conroy, S., O.N. Jarvis, G. Sadler and G.B. Huxtable (1988). Nucl. Fusion. 28, 2127.

Conroy, S., O.N. Jarvis, M. Pillon and G. Sadler (1990). Proc. 17th Eur. Conf. on Contr. Fusion and Plasma Heating, Amsterdam, 1990, Vol. 14B, Part 1, p. 98.

Cordey, J.G. et al. (1991). Proc. 18th Eur. Conf. on Contr. Fusion and Plasma Phys., Berlin, 1991, Vol. 15C, Part III, p. 385.

'Giannella. R.etal. (1991).Proc.18th Eur.Conf.on Contr.FusionandPlasmaPhys.,Berlin,1991, Vol.15C, PartI,p.197. 
Hinton, F.L. (1985). Nucl. Fusion 25, 1457.

Hogeweij, G.M.D., J. O'Rourke and A.C.C. Sips (1991). Plasma Phys. and Contr. Fusion 33, 189.

Jacquinot, J. and G. Sadler (1991), to be published in a special issue on D. ${ }^{3}$ He fusion of Fusion Technology and Design. Jarvis, O.N. et al. (1991). Proc. 18th Eur. Conf. on Contr. Fusion and Plasma Phys., Berlin, 1991, Vol. 15C, Part I,p.21. Jones, T.T.C., et al. (1991). Proc. I8th Eur. Conf. on Contr. Fusion and Plasma Phys., Berlin, 1991, Vol. 15C, Part I, p. 385 .

Keilhacker, M. and the JET Team (1990). Phys. Fluids B 2, 1291.

Keilhacker, M., R. Simonini, A. Taroni and M.L. Watkins (1991). Nucl. Fusion 31, 535.

Keilhacker, M.etal. (1991).In PlasmaPhys. and Contr.Nucl.FusionRes. 1990 (Proc.13thInt.Conf. Washington), Vol. 1, p. 345 (IAEA, Vienna, 1991).

Kupschus, P. et al. (1991). Proc. 18th Eur. Conf. on Contr.Fusion and Plasma Phys., Berlin.1991, Vol. 15C, Part I, p.1. Lackner, K. et al. (1984). Plasma Phys. and Contr. Fusion 26, 105 (1984).

Lomas, P.et al. (1991). Proc. I8th Eur. Conf. on Contr.Fusion and Plasma Phys., Berlin, 199l, Vol. 15C, Part I, p. 13. Lopes Cardozo, N.J. and A.C.C. Sips (1991), to be published in Plasma Phys. and Contr. Fusion.

Morgan, P. et al. (1991). Proc. 18th Eur. Conf. on Contr.Fusionand Plasma Phys.,Berlin, 199l, Vol. 15C, Part I, p. 361. Nardone, C. et al. (1991). Proc. 18th Eur. Conf. on Contr. Fusion and Plasma Phys., Berlin, 1991, Vol. 15C, Part I, p. 377.

O'Rourke, J.et al. (1991). Proc. I 8th Eur. Conf. on Contr. Fusion andPlasma Phys.,Berlin, 1991, Vol. 15C, Part I, p.37. Pasini, D. et al. (1991). Proc. I8th Eur. Conf. on Contr. Fusion and Plasma Phys., Berlin, 1991, Vol. 15C, Part I, p. 33. Rebut, P.H. et al. (1989). In: Plasma Phys. and Contr. Nucl. Fusion Res. 1988 (Proc. 12th Int. Conf. Nice), Vol. 2, p. 191 (IAEA, Vienna, 1989).

Reichle, R.etal. (1991).Proc.18thEur. Conf.on Contr.Fusionand PlasmaPhys.,Berlin, 1991, Vol.15C,Part III, p.105. Reiter, D., G.H. Wolf and H. Kever (1990). Nucl. Fusion 30, 2141.

Sadler, G. et al. (1991). Proc. 18th Eur. Conf. on Contr. Fusion and Plasma Phys., Berlin, 1991, Vol. 15C, Part I, p. 29. Schmidt, G.L. and the JETTeam (1989).In:Plasma Phys. and Contr.Nucl.FusionRes. 1988(Proc. 12th Int. Conf. Nice), Vol. 1, p. 215 (IAEA, Vienna, 1989)

Sips,A.C.C.etal. (1991).Proc. 18th Eur.Conf.on Contr.Fusionand Plasma Phys.,Berlin, 1991, Vol. 15C, Part I, p.193. Smetiders, P. eial. (1991).Proc. I8thEur. Conf.on Contr. FusionandPlasma Phys., Berlin, 1991, Vol.15C, Part I,p.53. Stambaugh, R.D. et al. (1991).In: Plasma Phys. and Contr. Nucl. Fusion . Res. 1990 (Proc. 13th Int. Conf. Washington), Paper CN-53/A-1-4.

Stork, D. et al. (1991). Proc. /8th Eur. Conf. on Contr. Fusion and Plasma Phys., Berlin, 1991, Vol. 15C, Part I, p. 357. Stubberfield, P., B. Balet and J. Cordey (1991). Plasma Phys. and Contr. Fusion 33.

Taroni, A. et al. (1991). In: Plasma Phys. and Contr. Nucl. Fusion Res. 1990 (Proc. 13th Int. Conf. Washington), Vol. 1, p. 93 (IAEA, Vienna, 1991)

Taroni, A., Ch. Sack, E. Springmann and $\overline{\mathrm{F}}$. Tibone (1991). Proc. 18 th Eur. Conf. on Contr. Fusion and Plasma Phys., Berlin, 199I, Vol. 15C, Part I, p. 181.

The JET Team, presented by J. Jacquinot (1991), this conference.

Tubbing, B.J.D. et al., to be published in Nucl. Fusion

Vlases, G. and R. Simonini (1991), Proc. 18th Eur. Conf. on Contr. Fusion and Plasma Phys., Berlin, 1991, Vol. 15C, Part III, p. 221.

Wagner, F. et al. (1991).In: Plasma Phys. and Contr. Nucl. Fusion. Res. 1990 (Proc. 13th Int. Conf. Washington), Paper CN-53/A-4-2.

Ward, D. et al. (1991). Proc. 18th Eur. Conf. on Contr.Fusion and Plasma Phys.,Berlin.1991, Vol. 15C, Part I, p. 353.

Watkins, M.L. et al. (1989). Plasma Phys. and Contr. Fusion 31, 1713 (Special Issue: Contr. Fusion and Plasma Phys., I6th Eur. Conf. of Eur. Phys. Soc., Plasma Phys. Div., Venice, 1989). 


\section{Appendix I \\ THE JET TEAM \\ JET Joint Undertaking, Abingdon, Oxon, OX14 3EA, U.K.}

J.M. Adams ${ }^{1}$,H. Almann, A. Andersen 14,P. Andrew18, M. Angelone29, S.A. Arshad, W. Bailey,P. Ballantyne, B. Balet, P. Barabaschi, R. Barnsley ${ }^{2}$, M. Baronian, D.V. Bartlett, A.C. Bell, I. Benfatto5, G. Benali, H. Bergsaker ${ }^{11}$, P. Bertoldi, E. Bertolini, V. Bhatnagar, AJ. Bickley, H. Bindslev 14, T. Bonicelli, S.J. Booth, G. Bosia, M. Botman, D. Boucher, P. Bouoquey, P. Breger, H. Brelen, H. Brinkschulte, T. Brown, M. Brusati, T. Budd, M. Bures, T. Businaro, P. Butcher, H. Buttgereit, C. Caldwell-Nichols, D.J.Campbell, P. Card, G. Celentano, C.D. Challis, A.V.Chankin 23, D. Chiron, J. Christiansen, C. Christodoulopoulos, P. Chuilon, R. Claesen, S. Clemert, E. Clipsham, J.P. Coad, M. Comiskey 4, S. Conroy, M. Cooke, S. Cooper, J.G. Condey, W. Core, G. Corrigan, S. Corti, A.E. Costley, G. Cotrell, M. Cox 7 , P. Cripwell, H. de Blank15, H. de Esch, L de Kock, E. Deksnis, G.B. Denne-Hinnov, G. Deschamps, KJ. Dietz, S.L. Dmitrenko,J. Dobbing, N. Dolgetta, S.E. Dorling,P.G. Doyle, D.F. Düctss,H. Duquenoy,A. Edwards,J. Ehrenberg, A. Ekedahl, T. Elevant 11 , S.K. Erents7, LG. Eriksson, H. Fajeminokun 12, H. Falter, D. Fory, J. Freiling 15, C. Froger, P. Froissard, K. Fullard,M. Gadeberg,A. Galetsas,D. Gambier,M. Garribba,P. Gaze, R. Giannella, A. Gibson,R.D. Gill, A. Girard, A. Gondhalekar, C. Gormezano, N.A. Gottandi, C. Gowers, B.J. Green, R. Haange, G Haas, A. Haigh, G. Hammett, CJ. Hancock, P.J. Harbour, N.C. Hawkes7, P. Haynes7, J.L Hemmerich, T. Hender?, F.B. Herzog, R.F. Herzog, J. Hoekzema, J. How, M. Huart, I Hughes, T.P. Hughes 4, M. Hugon, M. Huguet, A. Hwang7, B. Ingram, M. Irving, J. Jacquinot, H. Jaeckel, J.F. Jaeger, G. Janeschitz ${ }^{13}$, S. Jankowicz 22 , O.N. Jarvis, F. Jensen, E.M. Jones, L.P.D.F. Jones, T.T.C. Jones, J-F. Junger, E. Junique, A. Kaye, B.E. Keen, M. Keilhacker, G.J. Kelly, W. Kemer,

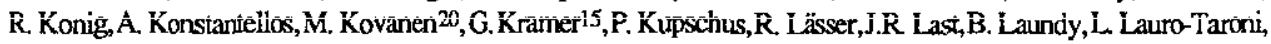
K. Lawson 7,M. Lenrholm, A. Loarte, R. Lobel, P. Lomas,M. Loughlin, C. Lowry, B. Macklin, G. Maddison 7, G. Magyar, W. Mandl13, V. Marchese, F. Marcus, J. Mart, E. Martin, R. Martin-Solis8, P. Massmann, G. MoCracken 7, P. Meriguet, P. Miele, S.F. Mills, P. Millward, R. Mohanti 17, P.L Mondino, A. Montvai'3 , S. Moriyama 28, P. Morgan, H. Morsi, G. Murphy,M. Mynarends, R Mymäs ${ }^{16}$,C. Nardone,F. Nave21,G. Newbert,M. Newman,P. Nielsen,P. Noll, W. Obert, D. O'Brien,J. O'Rourke, R. Ostrom,M Ottaviani,M. Pain,F. Paoletti,S. Papastergiou,D. Pasini,A. Peacock,N. Peacock 7, D. Pearson 12, R. Pepe de Silva, G. Perinic, C. Perry, M. Pick, R. Pitts 7, J. Plancoulkine, J-P. Poffe, F. Porcelli, L. Ponte ${ }^{19}$, R. Prentice, S. Puppin, S. Putvinsko'23, G. Radford9, T. Raimondi, M.C. Ramos de Andrade, P-H. Rebut, R. Reichle, E. Righi, F. Rimini, D. Robinson ${ }^{7}$, A. Rolfe, R.T. Ross, L. Rossi, R. Russ, P. Rutter, H.C. Sack, G. Sadler, G. Saibene, J.L. Salanave, G. Sanazzaro, A. Santagiustina, R. Sartori, C. Sborchia, P. Schild, M. Schmid, G. Schmidt 6 , B. Schunke, S.M. Scott, A. Sibley, R. Simonini, A.C.C. Sips, P. Smeulders, R. Stankiewicz 27, M. Stamp, P. Stangeby 18, D.F. Start, C.A. Steed, D. Stork, P.E. Stot, T.E. Stringer, P. Stubberfield, D. Summers, H. Summers ${ }^{19}$, L Svensson, J.A. Tagle2!, A. Tanga,A. Taroni,A. Tesini,P.R. Thomas,E. Thompson,K Thomsen,J.M. Todd,P. Trevalion,B. Tubbing,F. Tibone, E. Usselmann, H. van der Beken, G. Vlases,M. von Hellermann, T. Wade,C. Walker, R. Walton ${ }^{6}$, D. Ward,M.L. Watkins, MJ. Watson, S. Weber10, J. Wesson, T.J. Wijnands, J. Wilks, D. Wilson, T. Winkel, R. Wolf, B. Wolle ${ }^{24}$, D. Wong, C. Woodward, Y. Wu25, M. Wykes, ID. Young, L. Zannelli, Y. Zhu'25, W. Zwingmann.

\section{PERMANENT ADDRESSES}

1. UKAEA, Harwell, Didcot, Oxon, UK.

2. University of Leicester, Leicester, UK.

3. Central Research Institute for Physics, Acadeny of Sciences, Budapest, Hungary.

4. University of Essex, Colchester, UK.

5. ENEA-CNR, Padova, Italy.

6. Princeton Plasma Physics Laboratory, New Jersey, USA.

7. UKAEA Culham Laboratory, Abingdon, Oxon, UK.

8. Universidad Corrplutense de Madrid, Spain.

9. Institute of Mahematics, University of Oxford, UK.

10. Freien Universitäi, Berlin, F.R.G.

11. Swedish Energy Research Canrnission, S-10072 Stockholm, Sweden.

12. Imperial College of Science and Technology, University of London, UK.

13. Max Planck Institut für Plasmaphysik, Garching bei München, FRG.

14. Risø National Laboratory, Demmark.
15. FOM hnstibut voor Plasmafysica, 3430 Be Nieuwegein, The Netherlands.

16. University of Lund, Sweder.

17. North Carolina State University, Raleigh, NC, USA

18. Hstitutefor Aerospace Studies, University of Toronto, Downsview, Ontario, Canada

19. University of Strathclyde, 107 Rottenrow, Glasgow, UK.

20. Nuclear Engineering Laboratory, Lapeenranta University, Finland.

21. CIEMAT, Mactrid, Spain

22. Institute for Nuclear Sudies, Otwock-Swierk, Poland.

23. Kurchatov Institute of Atomic Energy, Moscow, USSR

24. University of Heidelberg, Heidelberg, FRG.

25. Instinute for Mechanics, Academia Sinica, Beijing, P.R. Ohina.

26. Southwestem University of Physics, Leshan, P.R. Chinz.

27. RCC Cyfronet, Otwock Swierk, Poland.

28. JAERL, Naka Fusion Research Establishment, Ibaraki, Japan.

29. ENEA, Frascati, italy. 